ReVISTA INTERnacional de Sociología (RIS)

Vol.68, No 3, SePTIEMBRE-DICIIEMBRE, 637-677, 2010

ISSN: 0034-9712

eISSN: 1988-429X

DOl:10.3989/ris.2008.10.17

\title{
EL RECUERDO DE VOTO EN ESPAÑA Un análisis del período 1996-2008
}

\author{
THE VOTE RECALL IN SPAIN \\ An Analysis of the Period 1996-2008
}

\author{
JAIME BALAguer \\ Centro de Investigaciones Sociológicas. CIS. España \\ jbalaguer@cis.es
}

\section{Resumen}

En este trabajo examinamos el recuerdo de voto recogido por el Barómetro del Centro de Investigaciones Sociológicas (CIS) a lo largo de tres legislaturas. Previamente, identificaremos las posibles fuentes de error que podrían sesgarlo. Nos detendremos en la influencia de las fallas en el proceso de recuperación, el sesgo de deseabilidad social y los efectos del instrumento de medición. Seguidamente daremos cuenta de sus principales sesgos a nivel agregado en España. Comprobaremos que el paso del tiempo introduce sucesivos errores en su declaración y, específicamente, veremos que parte de los entrevistados tendería a reconstruir su recuerdo de voto a partir de su intención de voto presente. Finalmente, prestaremos atención a la posible influencia de factores a corto plazo y al impacto del trabajo de campo. Nuestro objetivo es ayudar a los investigadores que emplean el recuerdo de voto a reconsiderar sus sesgos.

\section{Palabras Clave}

Efectos de orden, Encuestas electorales, Error de cobertura, Intención de voto, No respuesta, Trabajo de campo.

\begin{abstract}
In this paper we examine the evolution of the vote recall along three terms using the Barometer's data provided by the Center for Sociological Research (CIS, Spain). As a first step, we identify the potencial sources of error that could bias recall. Specifically, we will pay attention to the influence of mistakes in the retrieval process, in the social desirability bias and in the impact of the measurement tool. Secondly, we will show the main biases in Spain using aggregate data, showing that the passing of time introduces successive errors in voters' statements. Specifically, part of the interviewees tend to reconstruct their vote recall upon their present vote intention. Finally, we will see the possible influence of short-term factors and the impact of the fieldwork. Our goal is to help researchers who use the vote recall to rethink the biases.
\end{abstract}

\section{KEYWORDS}

Coverage Error, Electoral Surveys, Fieldwork, Nonresponse, Order effects, Vote Intention. 


\section{INTRODUCCIÓN ${ }^{1}$}

Tradicionalmente la investigación social ha enfrentado dificultades al intentar obtener de los ciudadanos respuestas precisas sobre temas como la sexualidad, la religión, la política o los ingresos. En el caso de las encuestas políticas, al preguntar por el voto en una elección pasada, obtenemos una variable conocida como "recuerdo de voto". Esta variable constituye la principal fuente de información sobre el comportamiento electoral de los encuestados, siendo empleada también para estimar los apoyos partidarios y la fidelidad de los electores. En contraste con la confianza depositada, una mera observación de su evolución en el tiempo llama poderosamente la atención (gráfico 1). La evolución de las categorías de respuesta muestra importantes irregularidades; un comportamiento que nos lleva a cuestionar su fiabilidad².

Si bien la pregunta por el voto pasado alude a una característica relativamente permanente y concreta de la población, el recuerdo del acto de votar se expresa de manera notablemente irregular. De manera que nos planteamos la siguiente paradoja: Si el voto en una elección pasada hace referencia a una característica estable de los entrevistados ¿Cómo se explica la variabilidad de su recuerdo?

A continuación examinaremos la evolución del recuerdo de voto durante tres legislaturas (1996-2008). La ausencia en España de estudios sistemáticos e intensivos parece haber consolidado una práctica investigadora ajena a consideraciones sobre sus sesgos, cuando una mera aproximación descriptiva evidencia anomalías que resulta necesario explicar. Gracias a las mediciones que, mes a mes y de manera homogénea, realiza el Barómetro del Centro de Investigaciones Sociológicas (CIS), tenemos una excelente oportunidad para analizar el caso español ${ }^{3}$.

Comenzaremos identificando exhaustivamente las fuentes de error que pueden afectar a la declaración del voto pasado. Partiendo de las evidencias de otros países plantearemos una serie de hipótesis para explicar su comportamiento en España. Dadas las particularidades de la información disponible, propondremos una estrategia de análisis basada en el empleo de datos agregados. De esta manera, constataremos que buena parte de su variabilidad puede ser explicada por factores ya identificados y que, además, sus sesgos pueden ser abordados satisfactoriamente desde esta perspectiva.

\footnotetext{
${ }^{1}$ Este manuscrito procede de la tesina del mismo título presentada en 2008 en el Máster en Democracia y Gobierno de la Universidad Autónoma de Madrid (UAM), siendo el tutor José Ramón Montero. Quiero expresar mi agradecimiento por sus revisiones y aportaciones a Ignacio Lago (Universitat Pompeu Fabra), a Santiago Pellegrini (Universidad Carlos III de Madrid), a los evaluadores de la RIS y, especialmente, a Alberto Sanz (UAM), sin cuyo apoyo este trabajo no habría sido posible.

${ }^{2}$ Descriptivos en el Anexo: Tabla A1.

${ }^{3}$ Un estudio continuo, de periodicidad mensual, dedicado al examen de la opinión pública en temas de la actualidad social, económica y política.
} 
Gráfico 1.

Evolución del recuerdo de voto durante la VI, VII y VIII legislatura.

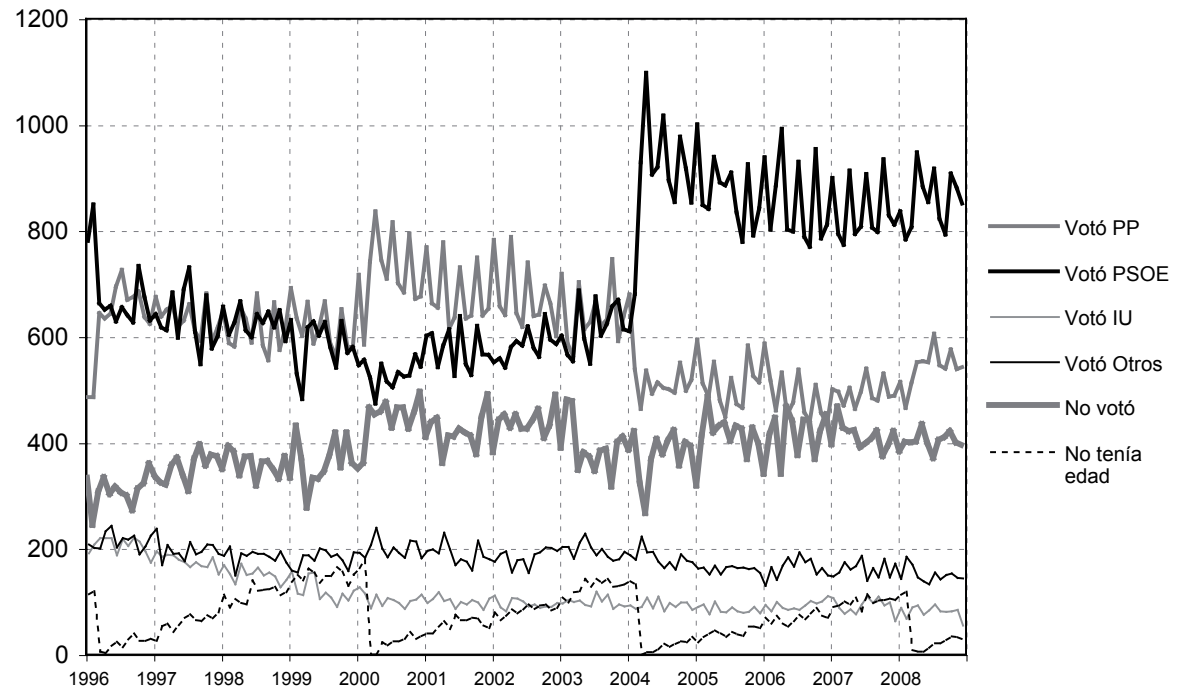

Fuente: Barómetro del CIS.

\section{LA CONTROVERSIA EN TORNO AL VOTO RECORDADO}

La distancia entre los resultados electorales oficiales y el voto recordado por los entrevistados ha llamado la atención desde el comienzo mismo de la difusión de encuestas políticas en los Estados Unidos, encontrándose una temprana evidencia de la presencia de sesgos sistemáticos (Dinerman 1949; Freeman 1953; Hugh y Crossley 1950; Miller 1952). Una de las obras clave de la ciencia política contemporánea, The American Voter, destaca la existencia de estos sesgos, enunciando las principales coordenadas del debate (Campbell et al. 1980: 93-96). En opinión de sus autores, las distorsiones estarían asociadas a tres causas fundamentales. En primer lugar, tendríamos efectos derivados de la metodología de encuesta; en segundo lugar, constataron una sobrestimación sistemática de la participación ocasionada por entrevistados que informaban erróneamente de su asistencia a las urnas (Parry y Crossley 1950). Finalmente, tendríamos el error en el recuerdo de la candidatura votada, un problema menor en términos agregados, al menos hasta el estudio del Survey Research Center de la Universidad de Michigan de las elecciones de 1964 (Clausen 1968). 
En torno a estas tres fuentes de error siguen estando, básicamente, las referencias con las que podemos estructurar el debate sobre los sesgos en el recuerdo de voto. A continuación, especificaremos las principales interpretaciones que se han dado del fenómeno para así encuadrar nuestro análisis de su comportamiento en el Barómetro del CIS.

\section{Sesgos en la medición de la participación}

La sobrestimación de la participación electoral es un fenómeno ampliamente contrastado, especialmente en los Estados Unidos. Desde 1948 ha estado siempre por encima del 5\%, aproximándose frecuentemente al 20\% (Abelson et al. 1992, citado por Stocké 2007; Adamany y Dubois 1975; Anderson y Silver 1986; Belli et al. 2001; Calahan 1968; Katosh y Traugott 1981; Mason, Lesser y Traugott 2002; Presser y Traugott 1992; Shaw et al. 2000; Sigelman 1982; Silver et al. 1986; Stocké y Stark 2007). Los análisis de este sesgo proceden en su mayoría de datos validados de los American National Election Studies, en los que se comprueba, mediante inspección del registro electoral, si los individuos que declaran haber votado en la encuesta realmente estaban inscritos y ejercieron su derecho al voto. El sesgo de participación es, posiblemente, el mejor estudiado, ya que este procedimiento permite estimar con relativa precisión el error a nivel individual. De esta manera sabemos que, en su práctica totalidad, la sobrestimación se debe a que un sector de abstencionistas declara haber votado cuando en realidad no lo ha hecho. El supuesto contrario, esto es, votantes reales que nieguen haberlo hecho, es tan reducido que podemos excluirlo del análisis.

Fuera del contexto estadounidense, los estudios de validación implementados arrojan resultados similares. En Reino Unido (Swaddle y Heath 1989), en Suecia (Andersson y Granberg 1997; Granberg y Holmberg 1991), en Noruega (Waldhal y Aardal 2000) y en Nueva Zelanda (Karp y Banducci 1999; Karp y Brockington 2005) se ha constatado que entre el $15-35 \%$ de los abstencionistas declararía erróneamente haber votado.

Para explicar la persistencia de este fenómeno los investigadores se remiten a dos causas principales, posiblemente interrelacionadas: el sesgo de deseabilidad social (SDS) y las fallas de la memoria. EI SDS hace referencia a una estrategia de autopresentación que aumenta la probabilidad de que los individuos informen de conductas y actitudes apropiadas y positivas. La información que entra en conflicto con esta autoimagen tiende a ser ignorada, rechazada o acomodada (Plumb 1986; Smith 1984). Parte de los individuos que no votaron declararía haberlo hecho al sentirse interpelados por el deber ciudadano de votar, al sentirse decepcionados ante sí mismos por no haber votado o para causar una buena impresión en los demás, poniendo de manifiesto el carácter sensible de esta pregunta (Tourengeau y Yan 2007). Diversas investigaciones suscribirían la intervención de este mecanismo (Duff et al. 2007; Holbrook et al. 2003; Holbrook y Krosnick 2010; Presser y Traugott 1992; Stocké 2007).

El contexto de la elección podría ejercer una influencia adicional sobre el SDS. Calahan (1968) ya había sugerido que cuantas más personas acudan a votar; mayor propor- 
ción de abstencionistas se verá inducida a recordar que votó. Karp y Brockington (2005), en un estudio comparado de cinco países encontraron que, efectivamente, tras comicios de alta participación, se incrementaba la proporción de recuerdo erróneo.

Por otra parte, diversas fallas de la memoria incidirían en un recuerdo de participación sesgado. Una de estas fallas refiere la recuperación asociada a una fuente errónea (Schacter 1999; Schacter y Dodson 2001). Como ya señalaba Calahan (1968), si el elector tiende normalmente a votar, asumirá que votó en todas las elecciones específicas que se le planteen. En nuestro caso operaría de la siguiente manera: interrogados sobre su conducta electoral, los entrevistados recurrirían a las representaciones disponibles en la memoria del acto de votar y, posteriormente, asociarán tales representaciones a la elección objeto de pregunta, por lo que el error será más probable si el entrevistado no ubica adecuadamente el evento de referencia (Kalton and Schuman 1982). De ahí también que diversos especialistas hayan identificado interferencias en el recuerdo de voto en elecciones nacionales vinculadas a la celebración posterior de elecciones regionales y locales (Belli et al. 2001; Waldhal y Aardal 2000).

Este error interactuaría con otra falla de la memoria, su fugacidad, que alude a la accesibilidad decreciente de la información conforme pasa en tiempo (Schacter 1999). Los errores resultarán más frecuentes cuanto más tiempo haya pasado desde el acto de votación, pues la conducta de referencia se encontrará más distante y menos accesible (Belli et al. 1999; Belli et al. 2001; Belli et al. 2006; Swaddle y Heath 1987). De hecho, Stocké y Stark (2007) han propuesto un mecanismo interactivo entre el SDS y la fugacidad: los incentivos para que actúe el SDS serán mayores cuanto más tiempo haya pasado y más dificultades tenga la memoria para recuperar el recuerdo.

Finalmente, será preciso considerar otra falla central: la parcialidad de la memoria. Con este término se alude a la probabilidad de distorsionar el recuerdo a partir de las creencias, sentimientos e informaciones del presente. La recuperación de contenidos tendería a exagerar la consistencia entre las actitudes presentes y pasadas (McDonald y Hirt 1997; Schacter 1999; Schacter, Chiao y Mitchell 2003). La influencia de este sesgo ha quedado en evidencia en diferentes estudios sobre el recuerdo de actitudes políticas (Joslyn 2003; Levine 1997; Marcus 1986). En nuestro caso implica que conforme se incremente el deseo de ir a votar, aumentará la probabilidad de recordar erróneamente haber votado en una elección pasada.

\section{Sesgos en el voto a las candidaturas}

Un segundo foco nos remite a los sesgos en la distribución del voto entre las candidaturas. Una mayoría de estudios constata un claro sesgo favorable a la candidatura ganadora, más acusado entre aquellos entrevistados que informaban erróneamente de su participación (Granberg y Holmberg 1986; Atkeson 1999; Wu 2006). Además, este sesgo resultará mayor cuanto más sobrepase la candidatura ganadora sus expectativas electorales previas (Katosh y Traugott 1981; Traugott y Katosh 1979; Wright 1990; Wright 1993). Otros analistas han cuestionado que el sesgo esté necesariamente vincu- 
lado al estatus de ganador (Clausen 1968). Específicamente, se han constatado sesgos favorables a los partidos mayoritarios (Benewick et al. 1969), a los que están en el poder (Himmelweit et al. 1978; Eubank y Gow 1983; Gow y Eubank 1984) y, finalmente, sesgos orientados hacia partidos específicos que resultarían sistemáticamente sobrerrepresentados o infraestimados (Gronke 1992; Moon 1994) ${ }^{4}$.

Las explicaciones a este fenómeno se han centrado en el miedo al aislamiento social y, nuevamente, en las fallas de la memoria. Respecto al primero, Wright (1990) señala que el sesgo favorable al ganador reflejaría un temor al aislamiento social en el sentido conceptualizado en La espiral del silencio por Noelle-Neumann (1995), de manera que ciertos entrevistados suprimirían sus expresiones de posiciones minoritarias basándose en su percepción de la opinión pública, mientras que otros tenderían a expresar sus propias preferencias al percibir que son mayoritarias (Scheufele y Moy 2000). Es más, si la derrota sufrida por el partido preferido es amplia - respecto a sus expectativas electorales previas - se acrecentaría esta sensación de vulnerabilidad, ya que justo tras la elección se configura un escenario político claramente favorable al ganador, más intenso cuanto mayor haya sido su éxito (Wright 1990; Wright 1993).

Las fallas de la memoria obrarían en un sentido similar al que vimos en el apartado anterior. Preguntados por su voto, los entrevistados buscarían en la memoria actos asociados al mismo. Estos recuerdos pueden existir porque se votó realmente, porque se hizo en otra elección diferente, porque se pensó en votar o porque se piensa votar en el futuro. Aquí, fidelidad e identificación partidaria resultan elementos críticos, pues ejercerían una función mnemotécnica, estabilizando el recuerdo. El error provendrá fundamentalmente de los electores con conductas de voto intermitentes y poco estructuradas; en ausencia de claves y de patrones estables con los que recuperar su recuerdo, tendrán mayor probabilidad de errar conforme pase el tiempo (Beckett et al. 2001; Himmelweit et al. 1978; Schaeffer y Presser 2003; Stocké y Stark 2007; Van Der Eijk y Niemöller 1983). Diferentes estudios de fiabilidad, mediante reentrevistas en las que se vuelve a preguntar a los mismos individuos por el sentido del voto pasado, han constatado una variabilidad en las respuestas superior al $15 \%$ en Estados Unidos, Reino Unido, Suecia, Noruega y Holanda (Benewick et al. 1969; Himmelweit et al. 1978; McDermind 1989; Niemi Katz y Newman 1980; Van Der Eijk y Niemöller 1983). Dicha variabilidad se incrementaría hasta el $40-60 \%$ entre los votantes swing -aquellos que cambiaron el sentido del voto entre dos comicios - y entre los que se han abstenido en alguna convocatoria (Waldahl y Aardal 1982).

Específicamente, en consonancia con los procesos de parcialización de la memoria, parte de los entrevistados tenderá a reconstruir su recuerdo a partir de sus valoraciones presentes, informando que votó por la candidatura por la que quiere votar. Diversos

\footnotetext{
${ }^{4}$ Urquizu (2005) constata una recurrente infraestimación de la intención de voto a determinados partidos españoles vinculada a su imagen, aunque la idea de un ocultamiento del voto, como veremos, podría resultar demasiado simplificadora.
} 
autores suscriben la intervención de este mecanismo (Atkeson 1999; Belli et al. 2001; Benewick et al. 1969; Granberg y Homlberg 1986; Himmelweit et al. 1978; McDermind 1989; Van Der Eijk y Niemöller 1983; Waldhal y Aardal 2000; Waldahl y Aardal 1982; Weir 1975; Wright 1993) ${ }^{5}$.

Finalmente, tal y como ocurría con el recuerdo de participación, se constata un efecto perturbador vinculado a la celebración posterior de las elecciones locales y regionales, ya que incrementan la probabilidad de recuperar un recuerdo empleando una representación errónea (Van Der Eijk 1983; Waldahl y Aardal 1982).

\section{Sesgos vinculados al proceso de medición}

Una tercera fuente de error la encontramos en el proceso de medición. En nuestro caso, deberemos considerar el impacto de la no respuesta - total o parcial- y del uso de procedimientos no probabilísticos en la selección de los entrevistados. La no respuesta parcial es originada por los entrevistados que han aceptado realizar la entrevista pero no han respondido directamente a nuestra variable de análisis. Este fenómeno adopta dos formas: como "No contesta" y "No recuerda". En términos generales, el "No contesta" -negativa a contestar al ítem- es más frecuente en preguntas sobre temas sensibles que no se desean compartir con extraños (Shoemaker et al. 2000) aunque, como ya señalamos, las preguntas sensibles también pueden estimular la aparición del SDS (Barnett 1998; Tourangeau y Yan 2007), no disponiéndose de evidencia que aclare en qué condiciones los entrevistados se inclinarían por una u otra opción.

En la pregunta por el voto pasado indagamos en un tema sensible, pues pretendemos que el entrevistado nos desvele un acto privado, individual y secreto, pudiendo despertar temor o recelo. Un recurso habitual para reducir la percepción amenazante de las preguntas sensibles consiste en ubicarlas al final del cuestionario, bajo la presunción de que un mayor tiempo de interacción y aproximaciones parciales previas al objeto irán reduciendo el temor del entrevistado (Bradburn et al. 2004); aunque este recurso se basa más en la práctica profesional que en estudios empíricos que lo respalden (Barnett 1998; Barton 1958; Tourangeau y Yan 2007). En nuestro caso, si durante la entrevista el encuestado va desvelando paulatinamente sus preferencias, valorando en primer lugar a los actores políticos, le resultará menos amenazante responder al final a preguntas más comprometidas como el recuerdo de voto. Así por ejemplo, en la mayoría de estudios electorales del CIS nuestra pregunta se ubica al final, después de declarar la intención de voto, lo que en principio reduciría su carácter amenazante. Pero también se introduce en encuestas sobre otros temas de actualidad social —relativamente alejados de la competición partidista - con la finalidad de caracterizar al entrevistado, antes del bloque de preguntas sociodemográficas. En este segundo tipo de encuestas, dado que no se producen

\footnotetext{
${ }^{5}$ La identificación partidaria estaría sometida a un proceso similar (Katz, Niemi y Newman 1980).
} 
aproximaciones previas a las preferencias partidarias, podemos suponer que la sensación de vulnerabilidad se incrementa, reflejándose en un aumento de las negativas a contestar.

Esto nos conduce directamente a la posible presencia de efectos de orden. Los efectos de orden se refieren a sesgos generados por las preguntas precedentes sobre un ítem, alterando sus patrones de relación. Los temas tratados en la encuesta generan un efecto de saliencia que afecta a las respuestas posteriores; hacen pensar en algo que es relevante para la siguiente cuestión -incluyendo, según Barnett (1998), la saliencia de las identidades grupales - incidiendo en el proceso de recuperación de información. Aunque no siempre se producen efectos de orden, su presencia ha sido confirmada en numerosos estudios de opinión pública (Duncan y Schuman 1977; Grice 1975; Kalton 1977; Noelle-Neumann 1970; Schuman y Presser 1996; Sigelman 1981), incluyendo las encuestas políticas (Bradburn y Mason 1964; Crespi y Morris 1984; Weisberg 2005), llegando a alterar los patrones de interés por el tema de la entrevista (McFarland 1981). De esta manera, se generarían expresiones de actitudes condicionadas, fundamentalmente sesgos de consistencia: las respuestas posteriores se situarían más próximas a las posiciones previas como resultado de su ordenación en el cuestionario (Bradburn y Mason 1964; Moore 2002; Schuman y Presse 1996; Sigelman 1981). En nuestro caso, en una entrevista en la que los individuos evalúan a los actores políticos -al llegar al final y plantear la pregunta por el voto pasado-, no sólo disminuirá el "No Contesta", sino que se podría estimular la expresión de un recuerdo consistente con las preferencias declaradas previamente. Se trata de un fenómeno diferente de la parcialidad de la memoria. Esta última operaría independientemente del tema y la ordenación de las preguntas en el cuestionario, mientras que el sesgo de consistencia refiere la influencia directa de las preguntas precedentes sobre nuestro ítem.

Por otra parte, las preguntas que requieren de un mayor esfuerzo cognitivo por parte del entrevistado, bien por su complejidad, bien porque indagan en eventos distantes del pasado, obtienen en mayor medida un "No sabe" o un "No recuerda", especialmente cuando el entrevistado carece de suficiente motivación (Shoemaker et al. 2000). Como hemos visto, la fugacidad de la memoria plantea un incremento sostenido en el tiempo del esfuerzo requerido para recuperar contenidos; así que, cuanto más distante esté la elección de referencia, obtendremos una mayor proporción de "No recuerda". De nuevo, los temas abordados previamente en la entrevista podrían alterar la probabilidad de obtener esta respuesta. El entrevistado necesita tener la información accesible, lo que depende de la relevancia que tenga el tema y del mismo cuestionario, que puede ofrecer claves que favorezcan la recuperación (Kalton y Schuman 1982). En nuestro caso, un cuestionario que solicita a los entrevistados evaluaciones de los actores políticos, incrementa la saliencia del tema (McFarland 1981), facilitando claves que apoyan el proceso de recuperación, con lo que la probabilidad de obtener un "No recuerda" descendería.

En otro orden, algunos autores han sugerido que parte de los sesgos en el recuerdo de voto obedecerían a problemas de cobertura, ocasionados por los entrevistados que rechazan la entrevista (Brehm 1993, citado por Jackman 1999; Swaddle y Heath 1987; Voogt y Van Kempen 2002; Voogt 2005). En concreto, la respuesta estaría sesgada por 
las personas más interesadas en la política, con un promedio de aceptación superior a la media (Groves et al. 2004; Weisberg 2005). Sin embargo, otros autores consideran que el error de cobertura no sería la causa de estas desviaciones, vista su consistencia en favorecer al ganador y su persistencia en muestras independientes, lo que apuntaría a una lógica diferenciada (Gow y Eubank 1984; Wright 1993).

Su potencial para distorsionar la representatividad de la muestra nos obliga a tomarlo seriamente en consideración. En su análisis de las incidencias del Barómetro, Núñez-Villuendas (2005) constata el crecimiento sostenido del número de intentos que los entrevistadores tienen que realizar para conseguir una entrevista. El problema más importante es el de las viviendas vacías ( $50 \%$ de las incidencias), destacando seguidamente los hogares que rechazan al entrevistador sin atender a ninguna explicación (9\%) y las personas seleccionadas que directamente rehúsan ser entrevistadas (10-12\%). Parte de estas incidencias se acrecentaría en las áreas urbanas y grandes ciudades, afectando de manera diferencial a determinados nichos geográficos, con lo que es probable que quede afectada la representatividad de sectores específicos del electorado, que también se distribuyen irregularmente en el territorio.

Finalmente, tenemos el problema del impacto del muestreo por cuotas en la selección última de los entrevistados, un procedimiento no probabilístico que también afecta a la representatividad de la muestra. En concreto, el Barómetro del CIS emplea el procedimiento de cuotas interdependientes de sexo y edad $^{6}$, predominante en las encuestas de opinión en España -y en países de nuestro entorno- y que pretende que la muestra resultante emule a la que se habría obtenido mediante procedimientos completamente probabilísticos, al menos en las variables empleadas para las cuotas. Precisamente Díaz de Rada y Núñez Villuendas (2008: 45-55) analizan la representatividad de las muestras del Barómetro del CIS con datos de la Encuesta de Población Activa (EPA) del Instituto Nacional de Estadística (INE), basada en un muestreo completamente probabilístico. Encontraron que el Barómetro sobrerrepresenta ligeramente a los desempleados, trabajadores por cuenta propia y del sector privado, aunque llama la atención la baja magnitud de los desajustes. Sin embargo, la aparente representatividad obtenida en unas variables no implica que se produzca en las restantes (Hochstim 1948; Kruskal 1980; Moser 1953), incluida la representatividad sociopolítica de la muestra (Lynn y Jowell 1996; Moon 1994; Smith 1983), ya que los grupos de sexo y edad no tienen un comportamiento electoral internamente homogéneo, ni por supuesto, justifica el empleo de un procedimiento no probabilístico. Además, sabemos que el muestreo por cuotas tiende a infrarrepresentar, entre otros grupos, a las personas más difíciles de localizar, a las más reacias a la entrevista, a las pertenecientes a grupos extremos en ingresos y educación y a las que viven en hogares de menor tamaño (Lynn 1995; Stephenson 1979; Sudman 1966).

\footnotetext{
${ }^{6}$ Con procedimientos probabilísticos en la selección de unidades primarias (municipios) y secundarias (secciones), así como un procedimiento de rutas aleatorias con instrucciones precisas para la selección de viviendas.
} 
Tanto los problemas de participación en la encuesta como el empleo de cuotas afectan a la representación de sectores sociales específicos, pudiendo reflejarse en una distribución de del recuerdo de voto sesgada. Sin embargo, si algo hemos aprendido de los estudios de validación y fiabilidad es que determinados sesgos operan de manera independiente (como la declaración errónea de participación y los sesgos vinculados al orden de las preguntas, a la celebración de elecciones regionales y locales 0 al cambio en las preferencias electorales). Como veremos, el examen de las incidencias de campo, mes a mes, nos permitirá controlar su potencial impacto.

\section{ESTRATEGIA DE ANÁLISIS E HIPÓTESIS}

Para analizar el recuerdo de voto en España emplearemos datos agregados procedentes del Barómetro del CIS. Una perspectiva adecuada - que no óptima- para nuestro objeto de estudio, ya que si bien no podremos establecer el nivel de error individual, la presencia de sesgos a nivel agregado - y su evolución en el tiempo-implica que los errores individuales no se han compensado entre sí y que tratamos con errores sistemáticos que apuntan en direcciones concretas (Anderson y Silver 1986; Stocké y Stark 2007).

Para establecer en qué medida el recuerdo es reconstruido sesgadamente, necesitamos dar cuenta de la estabilidad o constancia de la observación y compararla con un criterio independiente, en este caso, con los datos electorales oficiales. Será preciso establecer una aproximación adecuada a los parámetros reales del voto pasado de la población para cada mes en que se implementa el Barómetro. A partir de los datos del Ministerio del Interior sobre los resultados oficiales en elecciones al Congreso de los Diputados (para residentes en España) y del INE sobre incorporación de nuevos electores, hemos calculado qué recuerdo de voto deberíamos haber encontrado mensualmente en cada encuesta del Barómetro si los entrevistados tuviesen un recuerdo perfecto y totalmente ajustado a su conducta real. A esta aproximación la denominaremos "Modelo Esperado" (gráfico 2).

Como puede observarse, las diversas opciones experimentan saltos de nivel vinculados a la celebración de elecciones generales en 2000 y 2004, así como pendientes negativas - diferentes para cada legislatura-, debidas al impacto de la progresiva incoporación de nuevos votantes y a las bajas en el censo. A partir de la comparación de este modelo con el recuerdo recogido en el Barómetro estableceremos el alcance y sentido de sus principales sesgos. De esta manera, contrastaremos si:

H1: Tal y como hemos postulado sobre la intervención del SDS, encontraremos una sistemática infraestimación de la abstención, que además:

H1a: Será más acusada tras comicios con altos niveles de asistencia a las urnas, pues cuanto más mayoritaria haya sido la conducta de votar, mayores incentivos tendrán los abstencionistas para declarar que votaron. 
Gráfico 2.

Representación del Modelo Esperado del recuerdo de voto: 1996-2008.

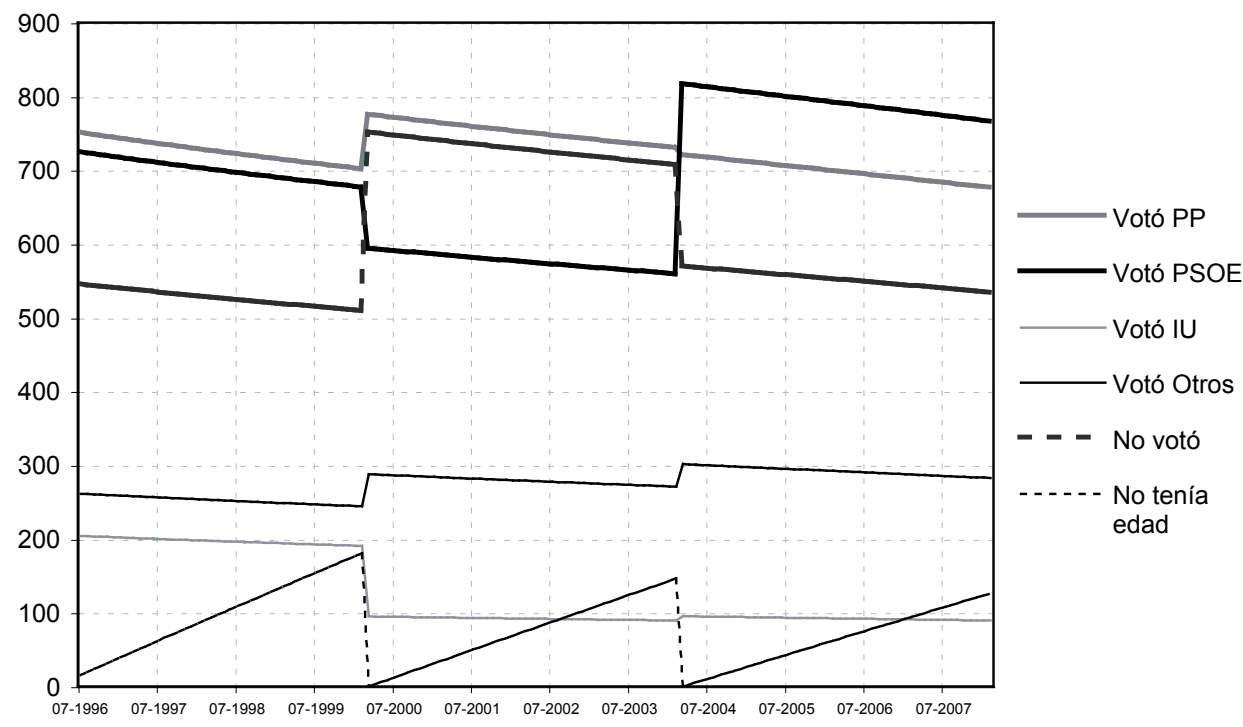

Fuente: Elaboración propia a partir de datos del Ministerio del Interior y del INE.

H1b: Se agravará cuanto más tiempo separe la entrevista del día de la elección de referencia, ya que se incrementan las dificultades de la memoria para recuperar el recuerdo y, por tanto, mayor probabilidad de que intervenga el SDS.

H2: Debido al miedo al aislamiento social, el sesgo en la distribución del voto será favorable a la candidatura ganadora, tanto más acusado cuanto mayor fuese su margen de victoria respecto a sus expectativas electorales previas; y tanto más desfavorable para el perdedor cuanto peores fuesen sus resultados respecto a lo esperado.

H3: Esperamos encontrar efectos relacionados con la celebración de elecciones municipales y autonómicas, debido a que incrementan la probabilidad de recuperar un recuerdo vinculado a una fuente errónea.

H4: En consonancia con lo planteado sobre la parcialización de la memoria, la evolución del recuerdo estará sesgada por los cambios en la intención de voto. Pero además:

H4a. En las encuestas en las que primero se evalúa a los actores políticos y se pregunta por la intención de voto este fenómeno se agravará, ya que estimulan la 
aparición de un sesgo de consistencia vinculado a las preguntas formuladas previamente en la entrevista.

H5: Dado que las encuestas donde los entrevistados evalúan previamente a los actores políticos disminuyen el temor a declarar el voto pasado, encontraremos descensos del "No contesta". Además:

H5a: Estas encuestas proporcionan claves y estímulos que facilitan el proceso de recuperación, por lo que registrarán descensos en el "No recuerda".

H6: Finalmente, dado que conforme pasa el tiempo el esfuerzo para recuperar el recuerdo se incrementa, cuanto más distante esté la elección de referencia, obtendremos una mayor proporción de "No recuerda".

Antes de proceder con el análisis, deberemos realizar una serie de consideraciones sobre los datos disponibles que afectan a las hipótesis $4 a, 5$ y 5a, ya que el Barómetro del $\mathrm{CIS}$ tiene una particularidad muy relevante. Cuatro entrevistas al año tienen la finalidad de obtener evaluaciones sobre los actores políticos y determinar los apoyos partidarios - meses de enero, abril, julio y octubre-, con un bloque de preguntas sobre competición electoral que va desde la valoración de los principales líderes políticos a la simpatía partidista y la intención de voto. Este bloque se comenzó a preguntar con una periodicidad trimestral a partir de julio de 1996 - con algunos recortes en enero de 2000 y 2004, omitiéndose en su totalidad en enero de 2008. Además, se introdujo una importante modificación en la pregunta tras las elecciones generales de 2008. Para evitar interferencias vinculadas a estos cambios, limitaremos nuestro rango de análisis al período en que la pauta de interrogación fue relativamente estable. En total disponemos de 128 observaciones: desde julio de 1996 a febrero de 2008.

Por otra parte, hemos sometido los datos a algunas transformaciones. En primer lugar, el Barómetro no se implementa en los meses de agosto, así que hemos imputado los valores de este mes con el promedio de los meses adyacentes que no versan sobre la competición electoral - junio y septiembre - incrementando el número de observaciones hasta 140. En ningún caso haremos interpretaciones de los resultados de estos meses. Además, el tamaño teórico de la muestra del Barómetro es de 2.500 entrevistas. Dado que no siempre se alcanza, hemos ajustado de manera proporcional los datos para este tamaño ${ }^{7}$.

La formulación de la pregunta en el período de análisis es la siguiente: “¿Me podría decir a qué partido o coalición votó $U d$. en las últimas elecciones generales de (mes y año de la elección)?", ante la que se anota la respuesta espontánea del entrevistado.

\footnotetext{
${ }^{7}$ Las entrevistas realizadas han oscilado entre las 2425 (mayo de 2005) y 2500 (marzo de 2001), con un promedio de 2489 entrevistados por mes.
} 
Las categorías con las que se codifica son: las principales candidaturas, "No votó", "Votó en blanco", "No tenía edad para votar", "No contesta" y "No recuerda". Dado que no se formularon dos preguntas, una para determinar si el entrevistado votó y otra a qué candidatura, no disponemos de una categoría que nos permita analizar la participación electoral, así que centraremos nuestra atención en la abstención a través de la categoría "No votó".

\section{LOS RESULTADOS}

Para poder contrastar eficientemente el recuerdo de voto del Barómetro con el Modelo Esperado, analizaremos el primero empleando modelos de regresión lineal estimados mediante el procedimiento de mínimos cuadrados. Las variables independientes serán, en primer lugar, las principales características del Modelo Esperado: una constante, la pendiente para la VI legislatura, el salto de nivel para las elecciones generales de 2000; la nueva pendiente para la VII legislatura; el salto de nivel para las elecciones 2004; y la pendiente para la VIII legislatura.

En segundo lugar, incluimos variables dummy que darán cuenta del efecto de los barómetros trimestrales que contienen el bloque de preguntas sobre competición electoral (valor 1) -debido a que las diferentes fuerzas políticas han gozado de apoyos variables y se han alternado en el gobierno, incluimos una para cada período legislativo-. Además, para dar cuenta del sesgo específico del contexto postelectoral introducimos otra dummy para marzo-abril de 2000 y marzo-abril de 2004.

Finalmente, introducimos otras tres dummy para las elecciones locales y autonómicas de ámbito nacional celebradas, identificando con valor 1 el tramo entre su celebración y las siguientes elecciones generales: Locales 1999 (junio de 19998-febrero de 2000); Locales 2003 (mayo de 2003-febrero de 2004) y Locales 2007 (mayo de 2007-febrero de 2008). Su finalidad es determinar si dichas elecciones coinciden con cambios de nivel en el recuerdo de voto. De manera que el modelo formulado sería9:

\footnotetext{
${ }^{8}$ En junio de 1999 se celebraron simultáneamente elecciones locales y al Parlamento Europeo. Hemos descartado estimar el efecto de las europeas de junio de 2004, por situarse en el contexto postelectoral de marzo de 2004.

${ }^{9}$ Se han excluido otras posibilidades como la introducción de lags t-1 o t-3 o similares, ya que nuestro objetivo no es predecir, sino describir la evolución del recuerdo y compararla con la prevista en el Modelo Esperado, ajustándonos a un modelo determinista.
} 
Recuerdo de voto en el Barómetro $0_{t}=\beta_{0}+\beta_{1} \cdot \tau_{1}+\beta_{2} \cdot t_{2}+\beta_{3} \cdot t_{3}+\beta_{4} \cdot$ Generales $2000+\beta_{5} \cdot$ Generales $2004+\beta_{6}$-Efecto Postelectoral $2000+\beta_{7}$-Efecto Postelectoral $2004+\beta_{8}$-Encuestas electorales 1996$2000_{\mathrm{t}}+\beta_{9}$-Encuestas electorales 2000-2004 $+\beta_{10}$ 'Encuestas electorales 2004-2008 $+\beta_{11}$. Locales $1999_{\mathrm{t}}$ $+\beta_{12}$ 'Locales $2003_{\mathrm{t}}+\beta_{13} \cdot$ Locales $2007_{\mathrm{t}}+\mathrm{e}$

Tabla 1.

Modelos de evolución para las categorías de respuesta del recuerdo de voto.

\begin{tabular}{|c|c|c|c|c|c|c|c|}
\hline & & PP & PSOE & IU & No votó & $\begin{array}{c}\text { No } \\
\text { recuerda }\end{array}$ & $\begin{array}{c}\text { No } \\
\text { contesta }\end{array}$ \\
\hline & Constante & $576^{\star *}$ & $828^{* *}$ & $292^{* *}$ & $274^{* *}$ & 2 & $278^{* *}$ \\
\hline \multirow{3}{*}{$>\frac{\frac{\pi}{\frac{\pi}{5}}}{\frac{\pi}{0}}$} & Pendiente 1996-2000 & $-1,48^{* *}$ & $-2,07^{\star *}$ & $-2,15^{\star *}$ & $1,15^{\star *}$ & $2,69^{* *}$ & $-1,05^{\star}$ \\
\hline & Encuesta Electoral 1996-2000 & $67^{* *}$ & $47^{* *}$ & $12^{* *}$ & $-28^{* *}$ & $-10^{*}$ & $-100^{* *}$ \\
\hline & Locales 1999 & 16 & 0 & -8 & -5 & -4 & 16 \\
\hline \multirow{5}{*}{ 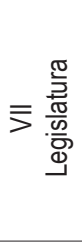 } & Elecciones 2000 & $202^{* *}$ & $-220^{\star *}$ & $-95^{\star *}$ & $127^{\star *}$ & $-64^{* *}$ & $176^{* *}$ \\
\hline & Efecto postelectoral 2000 & 32 & -41 & -3 & 29 & $-34^{* *}$ & 9 \\
\hline & Rec. pendiente 2000-2004 & $-1,43^{*}$ & $4,02^{* *}$ & $1,95^{\star \star}$ & $-1,30^{*}$ & $-0,92^{\star *}$ & $-1,88^{*}$ \\
\hline & Encuesta Electoral 2000-2004 & $106^{* *}$ & $28^{* *}$ & 4 & $-48^{* *}$ & $-14^{* *}$ & $-93^{* *}$ \\
\hline & Locales 2003 & 21 & 11 & 3 & $-52^{* *}$ & $-27^{* *}$ & $37^{*}$ \\
\hline \multirow{10}{*}{ 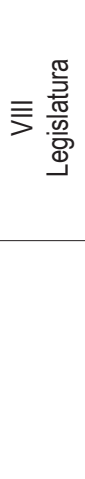 } & Elecciones 2004 & $-62^{* *}$ & $295^{* *}$ & -3 & $-48^{* *}$ & $-109^{* *}$ & $85^{* *}$ \\
\hline & Efecto postelectoral 2004 & -37 & $59^{*}$ & 8 & $-84^{* *}$ & -18 & 42 \\
\hline & Rec. pendiente 2004-2007 & $1,58^{* *}$ & $-5,17^{\star *}$ & 0,18 & $1,34^{*}$ & 0 & $1,97^{\star *}$ \\
\hline & Encuesta Electoral 2004-2007 & $58^{* *}$ & $116^{* *}$ & 4 & $-50^{* *}$ & $-11^{*}$ & $-130^{* *}$ \\
\hline & Locales 2007 & $26^{*}$ & $35^{\star}$ & 0 & $-37^{* *}$ & $-14^{*}$ & 8 \\
\hline & R cuadrado & 0,935 & 0,951 & 0,911 & 0,740 & 0,851 & 0,779 \\
\hline & R cuadrado corregido & 0,929 & 0,945 & 0,901 & 0,713 & 0,836 & 0,756 \\
\hline & Durbin-Watson & 1,51 & 2,01 & 1,82 & 1,55 & 1,74 & 1,82 \\
\hline & Estadístico F & $140,8^{* *}$ & $186,5^{\star *}$ & $98,7^{\star *}$ & $27,6^{\star *}$ & $55,3^{* *}$ & $34,1^{* *}$ \\
\hline & $\mathrm{N}$ & 140 & 140 & 140 & 140 & 140 & 140 \\
\hline
\end{tabular}

Fuente: Elaboración propia a partir del Barómetro del CIS.

${ }^{* *}$ Resultados significativos al 99\% ( $\left.p<0,01\right)$. ${ }^{*}$ Resultados significativos al 95\% $(p<0,05)$. 
En la tabla 1 tenemos sus resultados. Hemos preferido expresar los coeficientes en número de entrevistados en lugar de porcentajes, con el objeto de que las pendientes -que indican las tasas crecimiento mensual- no tengan demasiados decimales. Además, en la tabla no se expresan directamente las pendientes para 2000-2004 y 2004-2007, ya que los coeficientes refieren las rectificaciones de la pendiente del período anterior. Posteriormente desarrollaremos su contraste de manera más clara y específica.

Comprobamos que el ajuste global es relativamente bueno para las categorías de recuerdo de voto al PP, PSOE e IU ( $\mathrm{R}^{2}$ corregido>0,90), aunque no funciona tan bien para las categorías "No recuerda", "No contesta" y "No votó"10. Pero antes de avanzar, debemos realizar una aclaración. Dado que los modelos refieren la evolución de las series en tres legislaturas diferentes, con cambios de nivel para las elecciones de 2000 y 2004 , la interpretación de la constante $\left(\beta_{0}\right)$ no resulta evidente. A continuación examinaremos las discrepancias entre estos resultados y lo previsto por el Modelo Esperado.

\section{El recuerdo de la abstención ("No votó")}

En la tabla 2 hemos dispuesto el número de entrevistados con recuerdo "No votó" en el Barómetro - ajustado por el modelo de regresión y en sus niveles menores de "No contesta". En la columna adyacente tenemos los entrevistados que deberíamos haber encontrado según el Modelo Esperado, junto a su representación gráfica (Gráfico 3). Teniendo en cuenta el error muestral y las diferencias entre las pendientes, hemos identificado qué discrepancias resultan significativas.

En primer lugar, planteamos que encontraríamos una persistente infraestimación de la abstención (H1). Pues bien, la infraestimación de la abstención es profunda y afecta a todo el período analizado. Esta circunstancia pareciera confirmar la existencia de problemas para que los entrevistados reconozcan que no votaron. Sin embargo, si bien la presencia de falsos votantes es muy probable — debido al SDS - , parte del sesgo podría obedecer a un error de cobertura, que tendería a dejar fuera de la encuesta a los sectores menos interesados por la política.

En el contexto postelectoral, planteamos que la infraestimación de la abstención sería más acusada cuanto más alta hubiese sido la participación oficial (H1a). Aquí la evidencia confirmaría la propuesta. La infraestimación de la abstención en los meses de marzo y abril de 2004 — tras una elección de alta participación- es muy acusada, cosa que no ocurre en los mismos meses tras las elecciones de 2000, de menor participación.

Sin embargo, durante la VI y la VIII legislaturas la infraestimación de la abstención no se agudiza con el tiempo (H1b). De hecho, en estos dos períodos el recuerdo de abs-

\footnotetext{
${ }^{10}$ Según el test de Jarque-Vera los residuos presentan una distribución similar a la normal, excepto el "No contesta" que presenta más residuos positivos debido a que experimenta incrementos súbitos.
} 
Tabla 2 y Gráfico 3.

Contraste entre el Modelo esperado y el recuerdo de voto "No Votó".

\begin{tabular}{|c|c|c|c|}
\hline & & $\begin{array}{c}\text { Modelo } \\
\text { Esperado }\end{array}$ & $\begin{array}{c}\text { Barómetro } \\
\text { CIS }\end{array}$ \\
\hline \multirow{4}{*}{$\begin{array}{l}\text { VI } \\
\text { Legislatura }\end{array}$} & $\mathrm{N}$ al comienzo & 544 & $306^{*}$ \\
\hline & Pendiente & $-0,83$ & $1,15^{*}$ \\
\hline & Efecto locales 1999 & - & -5 \\
\hline & $\mathrm{N}$ al final & 508 & $350^{*}$ \\
\hline \multirow{5}{*}{$\begin{array}{l}\text { VII } \\
\text { Legislatura }\end{array}$} & $\mathrm{N}$ al comienzo & 750 & $403^{*}$ \\
\hline & Efecto postelectoral & - & 29 \\
\hline & Pendiente & $-0,93$ & $-0,15^{\star}$ \\
\hline & Efecto locales 2003 & - & $-52^{*}$ \\
\hline & $\mathrm{N}$ al final & 706 & $343^{*}$ \\
\hline \multirow{5}{*}{$\begin{array}{l}\text { VIII } \\
\text { Legislatura }\end{array}$} & $\mathrm{N}$ al comienzo & 568 & $351^{*}$ \\
\hline & Efecto postelectoral & - & $-84^{*}$ \\
\hline & Pendiente & $-0,74$ & $1,18^{*}$ \\
\hline & Efecto locales 2007 & - & -37 \\
\hline & $\mathrm{N}$ al final & 535 & $369^{*}$ \\
\hline
\end{tabular}

* La diferencia es significativa al 95,5\% ( $p<0,045)$

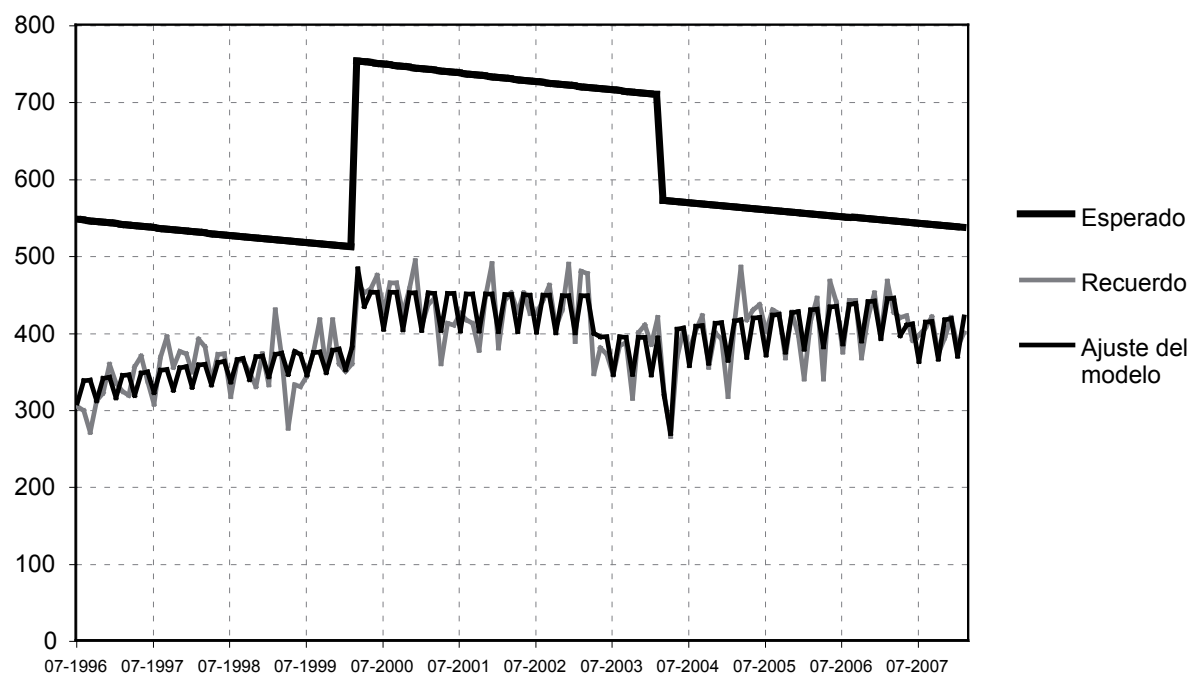

Fuente: Elaboración propia a partir del Barómetro del CIS, datos del Ministerio del Interior y del INE.

RIS, VOL.68. № 3, SEPTIEMBRE-DICIEMBRE, 637-677, 2010. ISSN: 0034-9712. DOI: 10.3989/ris.2008.10.17 
tención se incrementa progresivamente. Por tanto, el paso del tiempo no propiciaría una mayor intervención del SDS. Antes bien, esta peculiar evolución correspondería con una progresiva parcialización de la memoria $(\mathrm{H} 4)$, de manera que el crecimiento de recuerdo de haberse abstenido estaría reflejando un incremento progresivo de la intención de no votar.

Para ahondar en este punto, hemos seleccionado los barómetros trimestrales que incluyeron la pregunta sobre intención de voto y así estimar el coeficiente de correlación de Pearson entre el porcentaje de entrevistados con intención de abstenerse y el porcentaje de entrevistados con recuerdo "No votó". Ante esta prueba debemos de tomar precauciones, ya que los problemas de endogeneidad son evidentes: no podemos determinar si progresivamente hay más deseo de abstenerse o si en la muestra hay cada vez más abstencionistas aunque, como hemos argumentado, un crecimiento paulatino, mes a mes, de la presencia en la muestra de sectores específicos del electorado es poco probable. Además, al tener en cuenta sólo estos barómetros trimestrales el número de observaciones desciende a 44 -por lo que ofrecemos un único coeficiente. En este caso, arroja una relación positiva (gráfico 4) que se mantiene en los tres períodos considerados, lo que confirmaría la presencia de un sesgo dependiente de las motivaciones y evaluaciones del presente 0 , al menos, de una elevada consistencia entre el recuerdo y la intención de no votar.

Gráfico 4.

Correlación entre el porcentaje que declara haberse abstenido y el porcentaje que declara que se abstendrá en el futuro

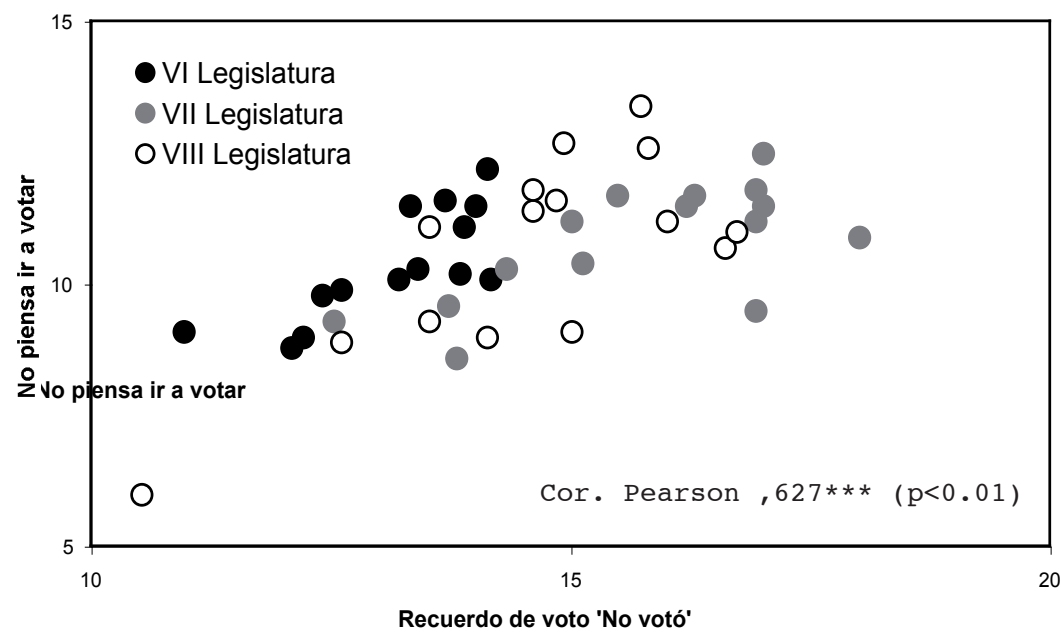

Fuente: Elaboración propia a partir del Barómetro del CIS. 
Finalmente esperábamos encontrar efectos asociados a la celebración de elecciones locales y autonómicas (H3). Aquí los resultados no son concluyentes. La celebración de elecciones en mayo de 2003 y mayo de 2007 sí que tuvieron efecto: se produjo una retirada efectiva del recuerdo "No votó", aunque desconocemos por qué no se presentó tras las locales de 1999.

\section{El recuerdo de voto a las candidaturas}

Por otra parte planteamos que, en el contexto postelectoral, el recuerdo para el partido ganador estaría tanto más sobrestimado cuanto mayor hubiese sido su margen de victoria respecto a sus expectativas $(\mathrm{H} 2)$. Complementariamente, el partido perdedor quedaría infraestimado, tanto más cuanto peores resultados haya obtenido. Para abordar este punto deberemos concretar antes cuáles eran las expectativas previas de los principales contendientes electorales, PP y PSOE. En la tabla 3 tenemos algunos datos relevantes: la intención de voto estimada por el CIS, el voto finalmente obtenido y, lo más importante, el porcentaje de entrevistados que creía ganador a una u otra formación. En las elecciones de 1996 la victoria del PP, aunque prevista, fue menor de la esperada, mientras que en 2000 fue mayor; por el contrario su derrota en 2004 ni fue prevista por las encuestas, ni era esperada por los electores.

Tabla 3.

Voto esperado frente a voto obtenido

\begin{tabular}{|c|c|c|c|c|c|c|c|c|c|}
\hline & \multicolumn{3}{|c|}{1996} & \multicolumn{3}{|c|}{2000} & \multicolumn{3}{|c|}{2004} \\
\hline & 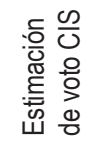 & 용 & 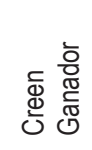 & 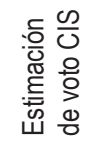 & 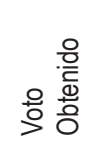 & 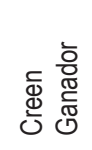 & 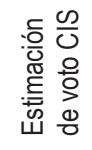 & 을 & 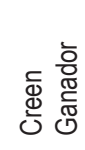 \\
\hline PP & $41,2 \%$ & $38,8 \%$ & $56,7 \%$ & $41,6 \%$ & $44,5 \%$ & $63,9 \%$ & $42,2 \%$ & $37,6 \%$ & $63,4 \%$ \\
\hline PSOE & $34,1 \%$ & $37,6 \%$ & $13,2 \%$ & $36,6 \%$ & $34,0 \%$ & $10,2 \%$ & $35,5 \%$ & $42,6 \%$ & $11,0 \%$ \\
\hline
\end{tabular}

Fuente: CIS2207, CIS2382 y CIS2555 y Ministerio del Interior.

Pues bien, aunque el efecto sólo resulta significativo para el PSOE en 2004 (tablas 4 y 5), los resultados apuntan en la dirección esperada: una infraestimación de los socialistas en 2000 y de los populares en 2004, así como una sobrestimación de los populares en 2000 y, sobre todo, del PSOE en 2004, que se corresponde con una intensa caída del recuerdo de abstención. De manera que las victorias y derrotas, y especialmente su contraste con las expectativas previas, determinarían el alcance de los sesgos en el contexto postelectoral. 
Tabla 4 y Gráfico 5.

Contraste entre el Modelo esperado y el recuerdo de voto al PP

\begin{tabular}{|c|c|c|c|}
\hline & & $\begin{array}{c}\text { Modelo } \\
\text { Esperado }\end{array}$ & $\begin{array}{c}\text { Barómetro } \\
\text { CIS }\end{array}$ \\
\hline \multirow{4}{*}{$\begin{array}{l}\text { VI } \\
\text { Legislatura }\end{array}$} & $\mathrm{N}$ al comienzo & 750 & $701^{*}$ \\
\hline & Pendiente & $-1,15$ & $-1,48$ \\
\hline & Efecto locales 1999 & - & 16 \\
\hline & $\mathrm{N}$ al final & 700 & $654^{*}$ \\
\hline \multirow{5}{*}{$\begin{array}{l}\text { VII } \\
\text { Legislatura }\end{array}$} & $\mathrm{N}$ al comienzo & 774 & 810 \\
\hline & Efecto postelectoral & - & 32 \\
\hline & Pendiente & $-0,96$ & $-2,92^{*}$ \\
\hline & Efecto locales 2003 & - & 21 \\
\hline & $\mathrm{N}$ al final & 729 & 694 \\
\hline \multirow{5}{*}{$\begin{array}{l}\text { VIII } \\
\text { Legislatura }\end{array}$} & $\mathrm{N}$ al comienzo & 720 & $564^{*}$ \\
\hline & Efecto postelectoral & - & -37 \\
\hline & Pendiente & $-0,94$ & $-1,33$ \\
\hline & Efecto locales 2007 & - & 26 \\
\hline & $\mathrm{N}$ al final & 677 & $528^{*}$ \\
\hline
\end{tabular}

* La diferencia es significativa al 95\% ( $p<0,045)$

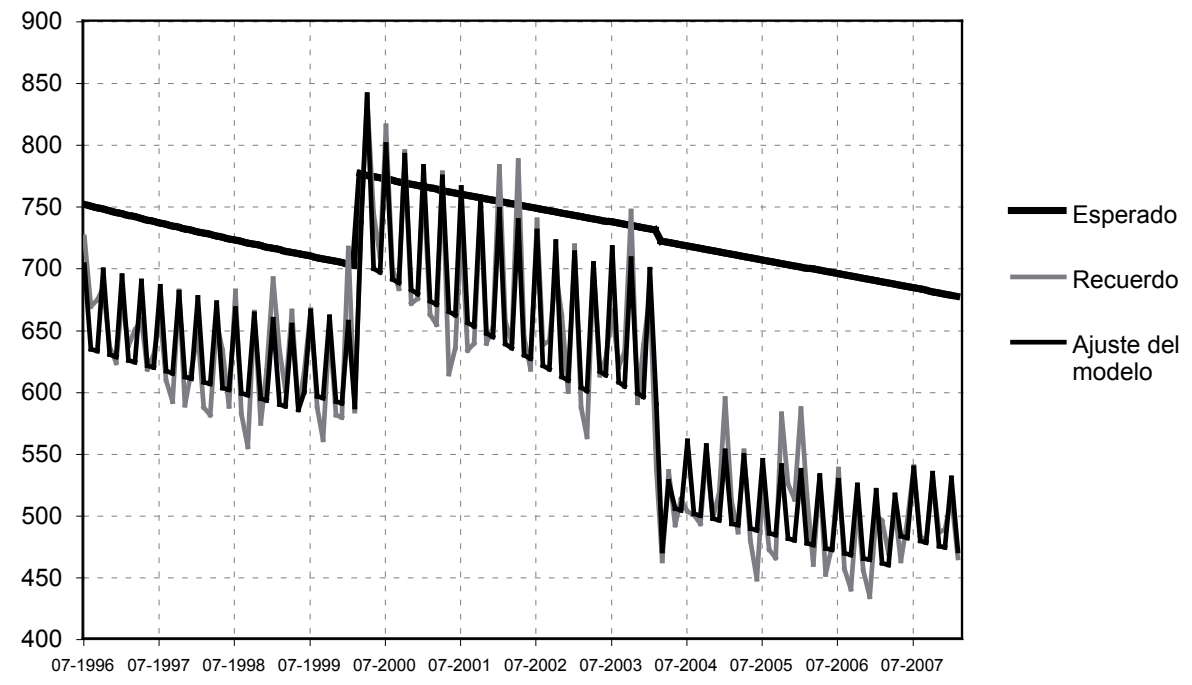

Fuente: Elaboración propia a partir del Barómetro del CIS, datos del Ministerio del Interior y del INE. 
Tabla 5 y Gráfico 6.

Contraste entre el Modelo esperado y el recuerdo de voto al PSOE

\begin{tabular}{|c|c|c|c|}
\hline & & $\begin{array}{c}\text { Modelo } \\
\text { Esperado }\end{array}$ & $\begin{array}{c}\text { Barómetro } \\
\text { CIS }\end{array}$ \\
\hline \multirow{4}{*}{$\begin{array}{l}\text { VI } \\
\text { Legislatura }\end{array}$} & $\mathrm{N}$ al comienzo & 723 & 692 \\
\hline & Pendiente & $-1,10$ & $-2,07^{*}$ \\
\hline & Efecto locales 1999 & - & 0 \\
\hline & $\mathrm{N}$ al final & 676 & $603^{*}$ \\
\hline \multirow{5}{*}{$\begin{array}{l}\text { VII } \\
\text { Legislatura }\end{array}$} & $\mathrm{N}$ al comienzo & 593 & $551^{*}$ \\
\hline & Efecto postelectoral & - & $-41^{*}$ \\
\hline & Pendiente & $-0,74$ & $1,96^{*}$ \\
\hline & Efecto locales 2003 & - & 11 \\
\hline & $\mathrm{N}$ al final & 558 & $655^{*}$ \\
\hline \multirow{5}{*}{$\begin{array}{l}\text { VIII } \\
\text { Legislatura }\end{array}$} & $\mathrm{N}$ al comienzo & 815 & $1012^{*}$ \\
\hline & Efecto postelectoral & - & $59^{*}$ \\
\hline & Pendiente & $-1,07$ & $-3,22^{*}$ \\
\hline & Efecto locales 2007 & - & 35 \\
\hline & $\mathrm{N}$ al final & 767 & $895^{*}$ \\
\hline
\end{tabular}

* La diferencia es significativa al 95,5\% ( $p<0,045)$

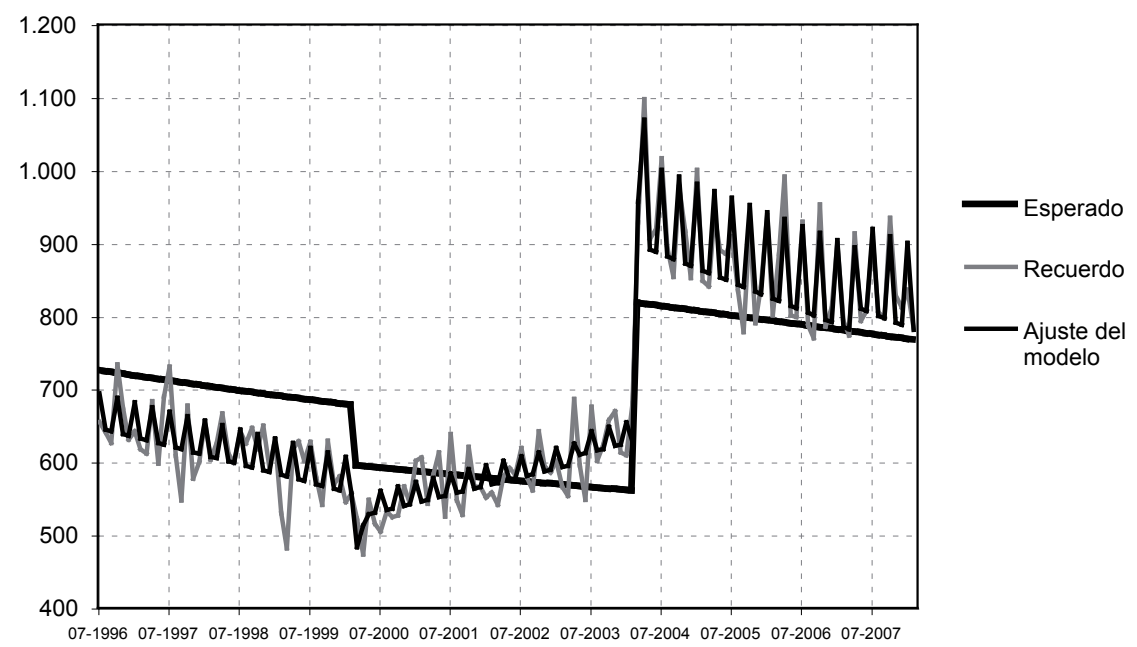

Fuente: Elaboración propia a partir del Barómetro del CIS, datos del Ministerio del Interior y del INE.

RIS, VOL.68. № 3, SEPTIEMBRE-DICIEMBRE, 637-677, 2010. ISSN: 0034-9712. DOI: 10.3989/ris.2008.10.17 
Fuera del contexto postelectoral, constatamos como persiste la infraestimación del voto popular durante la VI y la VIII legislaturas, así como una destacable sobrestimación del voto socialista en esta última. Pero aunque estos sesgos sean consistentes con lo previsto, no podemos aseverar que su origen esté en el miedo al aislamiento social. En primer lugar, podrían estar relacionados con problemas de participación en la encuesta. Por ejemplo, los electores que apoyaron al perdedor en 2004 podrían mostrar una mayor tendencia a rechazar la entrevista. Para descartar esta posibilidad hemos examinado las incidencias del Barómetro del CIS del período 2001-200711 que mayor probabilidad tienen de afectar a la representatividad de la muestra: las negativas y las viviendas vacías. Empleando nuestro modelo de regresión, comprobamos que ni la celebración de elecciones generales en 2004, ni los barómetros sobre competición electoral se corresponden con cambios en las incidencias ${ }^{12}$.

También podría suceder que el empleo de cuotas tuviese un impacto —renovado tras las elecciones de 2004 y la subsecuente recomposición del electorado- que perjudicara la representatividad del electorado popular. Sin embargo, este no es un problema específico del muestreo por cuotas. Las dos encuestas completamente probabilísticas del International Social Survey Programme-ISSP (Anexo: gráficos A1) obtienen resultados similares ${ }^{13}$, aunque sus importantes problemas de no respuesta podrían afectar de manera similar a estos electores.

En términos generales consideramos que el sesgo en la distribución del voto tiene una doble orientación: se orienta negativamente hacia un partido específico, el PP, y lo hace de manera persistente en todo el período. Pero además, se ve afectado por el alcance de las victorias y derrotas de los partidos, y este segundo fenómeno sí que puede vincularse con la espiral del silencio. La evolución en el tiempo expresada en las pendientes nos permite detectar anomalías importantes que matizan esta interpretación.

El recuerdo al PP en 2000-2004 experimenta un descenso superior al previsto: a un ritmo de -2,92 entrevistados/mes cuando esperábamos una pendiente de -0,96. También el recuerdo al PSOE en 1996-2000 desciende a un ritmo algo mayor de lo esperado, quedando infrarrepresentado para el final de la legislatura. Pero es en la legislatura 2000-2004 donde se produce la evolución más llamativa. El recuerdo al PSOE se incrementa progresivamente, con una pendiente de +1,96 - frente al -0,74 previsto-, contraviniendo uno de los supuestos del Modelo Esperado, que pronostica siempre una pendiente negativa para todas las opciones partidarias. Finalmente, en 2004-2007 el PSOE vuelve a evolucionar negativamente, aunque comienza y termina el período en niveles destacados de sobrerrepresentación. Estas evoluciones sesgadas se correspon-

\footnotetext{
${ }^{11}$ No disponemos de datos de incidencias adecuadamente desagregados anteriores a 2001.

${ }^{12}$ Resultados no mostrados.

${ }^{13}$ Las encuestas nominales de la European Social Survey (ESS) emplean dos preguntas para obtener el recuerdo de voto, por lo que no podemos establecer una comparación, aunque persiste en ellas la infraestimación del electorado popular.
} 
den nuevamente con un "recuerdo parcializado", reflejando los progresivos cambios en la intención de voto $(\mathrm{H} 4)$. Los coeficientes de correlación apuntan a una consistencia muy elevada entre el recuerdo y la intención de voto (Gráficos 7 y 8 ).

También el recuerdo de IU muestra una pauta similar (Tabla 6 y Gráfico 9). En 19962000 encontramos un descenso muy superior al esperado: una pendiente de $-2,15$ cuando se esperaba un descenso de $-0,31$ entrevistados/mes, de manera que, para finales de 1999, la coalición había perdido la mitad de su recuerdo de voto, quedando claramente infrarrepresentada.

Gráficos 7.

Correlación entre el porcentaje de recuerdo de voto PP y la intención de voto PP

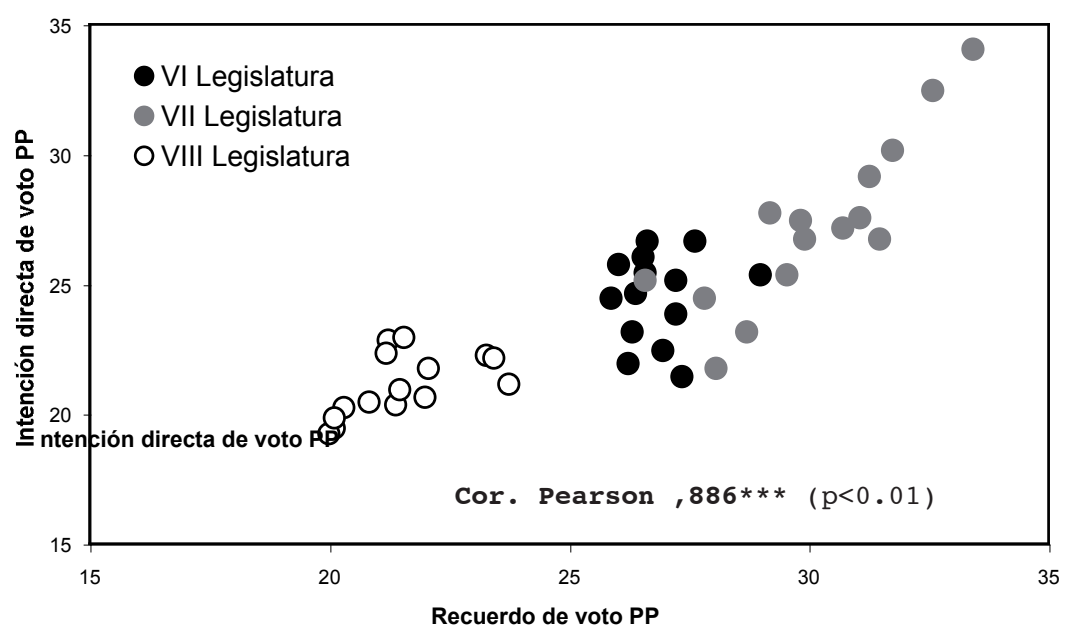

Fuente: Elaboración propia a partir del Barómetro del CIS. 
Gráficos 8.

Correlación entre el porcentaje de recuerdo de voto PSOE y la intención de voto PSOE

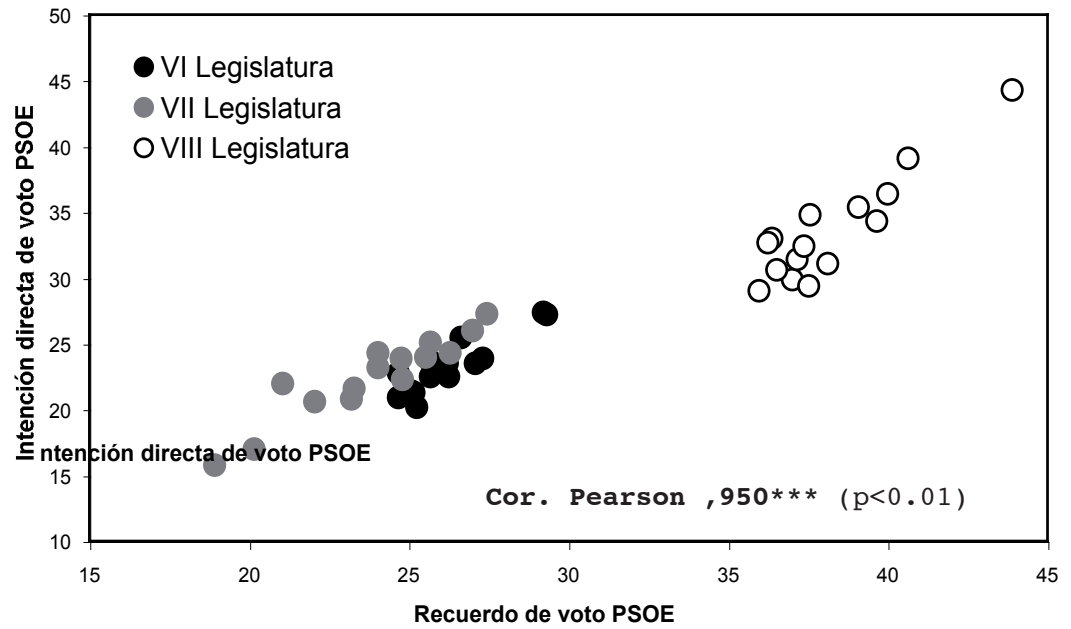

Fuente: Elaboración propia a partir del Barómetro del CIS.

Tabla 6.

Contraste entre el Modelo Esperado y el recuerdo de voto 'IU'

\begin{tabular}{|c|c|c|c|}
\hline & & $\begin{array}{c}\text { Modelo } \\
\text { Esperado }\end{array}$ & $\begin{array}{c}\text { Barómetro } \\
\text { CIS }\end{array}$ \\
\hline \multirow{4}{*}{$\begin{array}{l}\mathrm{VI} \\
\text { Legislatura }\end{array}$} & $\mathrm{N}$ al comienzo & 204 & 211 \\
\hline & Pendiente & $-0,31$ & $-2,15^{*}$ \\
\hline & Efecto locales 1999 & - & -8 \\
\hline & $\mathrm{N}$ al final & 191 & $110^{*}$ \\
\hline \multirow{5}{*}{ VII Legislatura } & $\mathrm{N}$ al comienzo & 95 & 104 \\
\hline & Efecto postelectoral & - & -3 \\
\hline & Pendiente & $-0,12$ & $-0,19$ \\
\hline & Efecto locales 2003 & - & 3 \\
\hline & $\mathrm{N}$ al final & 89 & 98 \\
\hline \multirow{5}{*}{$\begin{array}{l}\text { VIII } \\
\text { Legislatura }\end{array}$} & $\mathrm{N}$ al comienzo & 95 & 93 \\
\hline & Efecto postelectoral & - & 8 \\
\hline & Pendiente & $-0,12$ & $-0,15$ \\
\hline & Efecto locales 2007 & - & 0 \\
\hline & $\mathrm{N}$ al final & 90 & 92 \\
\hline
\end{tabular}

* La diferencia es significativa al 95,5\% ( $p<0,045)$ 
Gráfico 9.

Contraste entre el Modelo Esperado y el recuerdo de voto 'IU'

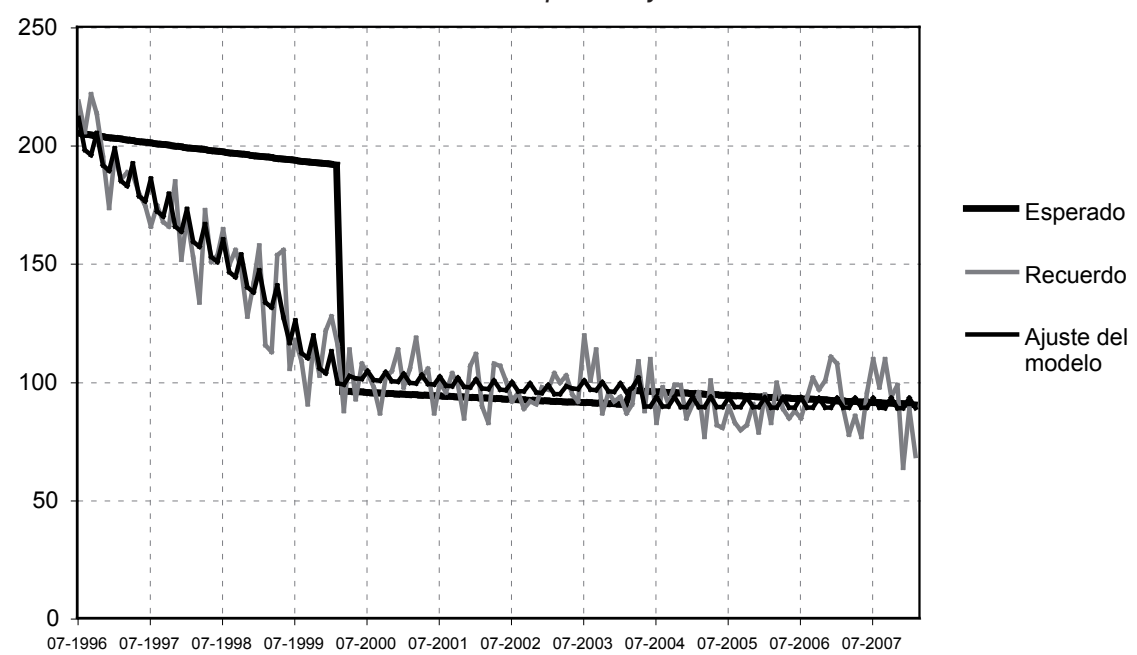

Fuente: Elaboración propia a partir de Barómetro del CIS, datos del Ministerio del Interior y del INE.

Esta evolución de IU coincide con una importante y sostenida pérdida de apoyos durante la legislatura 1996-2000, encontrándose una alta consistencia con los cambios en la intención de voto (Gráfico 10).

Gráfico 10.

Correlación entre el porcentaje de recuerdo de voto IU e intención de voto IU

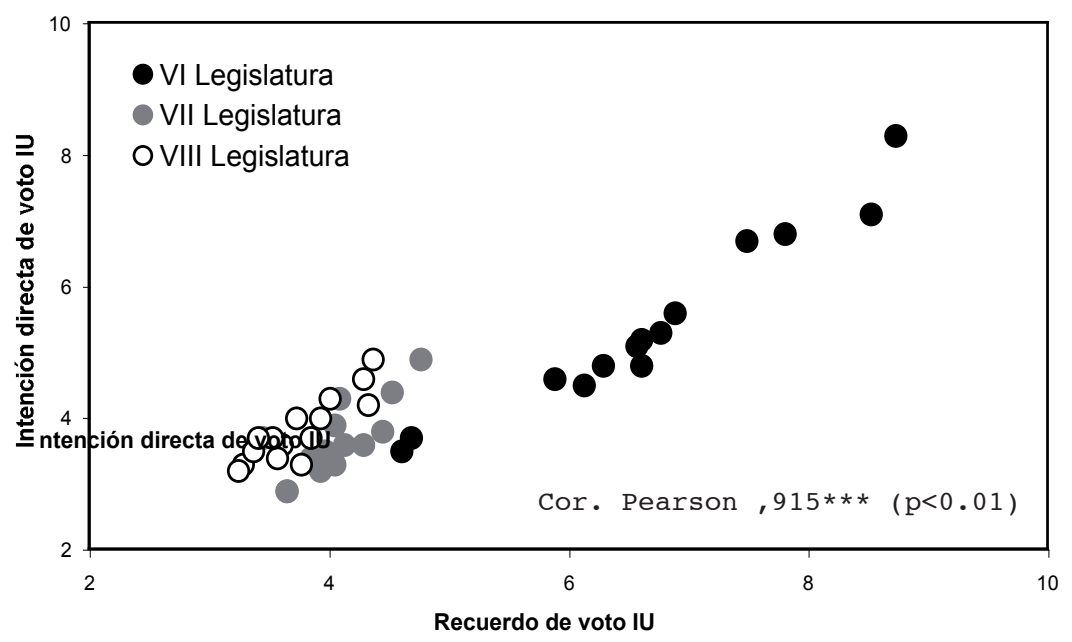

Fuente: Elaboración propia a partir del Barómetro del CIS.

RIS, VOL.68. No 3, SEPTIEMBRE-DICIEMBRE, 637-677, 2010. ISSN: 0034-9712. DOI: 10.3989/ris.2008.10.17 
Además de un recuerdo parcializado, nos planteamos la posible intervención de efectos de orden. Concretamente, en las encuestas electorales las preguntas previas podrían inducir la declaración de un recuerdo más consistente con lo expresado previamente en la entrevista ( $\mathrm{H} 4 \mathrm{a})$. Para establecer si los barómetros sobre competición electoral han ejercido una influencia excesiva en estas evoluciones sesgadas, hemos vuelto a calcular las pendientes, separadas según el tipo de Barómetro (tabla 7).

Tabla 7.

Contraste entre las pendientes en las encuestas con el bloque de competición electoral frente al resto de Barómetros

\begin{tabular}{|c|c|c|c|c|c|c|c|c|}
\hline & \multicolumn{2}{|c|}{ No votó } & \multicolumn{2}{|c|}{$\mathrm{PP}$} & \multicolumn{2}{|c|}{ PSOE } & \multicolumn{2}{|c|}{ IU } \\
\hline & $\begin{array}{c}\text { Enc. } \\
\text { Electoral }\end{array}$ & Resto & $\begin{array}{c}\text { Enc. } \\
\text { Electoral }\end{array}$ & Resto & $\begin{array}{c}\text { Enc. } \\
\text { Electoral }\end{array}$ & Resto & $\begin{array}{c}\text { Enc. } \\
\text { Electoral }\end{array}$ & Resto \\
\hline $1996-2000$ & $+1,35$ & $+0,31^{*}$ & 0,00 & $-1,60^{*}$ & $-2,81$ & $-1,80$ & $-2,12$ & $-2,43$ \\
\hline $2000-2004$ & $-1,71$ & $-1,19$ & $-2,94$ & $-2,50$ & $+3,21$ & $+1,95^{*}$ & $-0,19$ & $-0,09$ \\
\hline $2004-2008$ & $+1,50$ & $+0,61$ & $-0,78$ & $-0,56$ & $-3,34$ & $-2,60$ & $-0,02$ & $-0,08$ \\
\hline
\end{tabular}

* La diferencia es significativa al 95\% ( $p<0,05)$.

Fuente: Elaboración propia a partir del Barómetro del CIS.

La mayoría de las pendientes son similares, independientemente del tipo de barómetro. Por tanto, aunque la entrevista verse sobre temas alejados de la política, los entrevistados siguen declarando un recuerdo de voto progresivamente sesgado, lo que apunta a una genuina parcialización de la memoria y no a un simple efecto de las preguntas sobre competición electoral. Las excepciones las encontramos fundamentalmente en 19962000 , donde la pendiente negativa del PSOE en las encuestas de competición electoral es más acusada, mientras que la del PP es nula y la de la abstención crece a un ritmo todavía mayor. La otra diferencia la encontramos en 2000-2004, donde el recuerdo de voto al PSOE crece a un ritmo más intenso en estos barómetros electorales.

En cuanto a la interferencia de las elecciones locales y autonómicas $(\mathrm{H} 3)$ encontramos nuevamente que las de 1999 no indujeron ningún efecto. Las de 2003 sí que coinciden con una recuperación del recuerdo al PP, pero no del PSOE, probablemente porque en esa legislatura está experimentando una anormal progresión positiva. Las de 2007 en cambio, sí coinciden con una recuperación del recuerdo tanto del PP como del PSOE, mientras que el recuerdo de IU no se vio alterado en todo el período analizado. Estas expresiones parciales deben ser afrontadas. Su presencia podría depender de elementos específicos del contexto de la elección. Pero también podríamos haber incurrido en una operacionalización deficiente. El examen realizado con el recuerdo de voto 
recogido en Cataluña ${ }^{14}$ muestra que las elecciones al Parlament no tuvieron impacto en el recuerdo de voto en las generales. En definitiva, no parece que necesariamente vayamos a encontrar efectos vinculados a la celebración de otras elecciones, quedando pendiente un análisis más específico de en qué circunstancias pueden presentarse.

\section{La no respuesta parcial}

Nos planteamos dos hipótesis para el "No recuerda". Por un lado, apuntamos a que las encuestas electorales proporcionarían claves e incrementarían la motivación de los entrevistados para recuperar el recuerdo, encontrándonos con descensos en el "No recuerda" (H5a). Comprobamos que dichos descensos se producen, aunque su magnitud es muy reducida (Tabla 1 y Gráfico 11).

\section{Gráfico 11. \\ Modelo para el recuerdo de voto 'No Recuerda'}

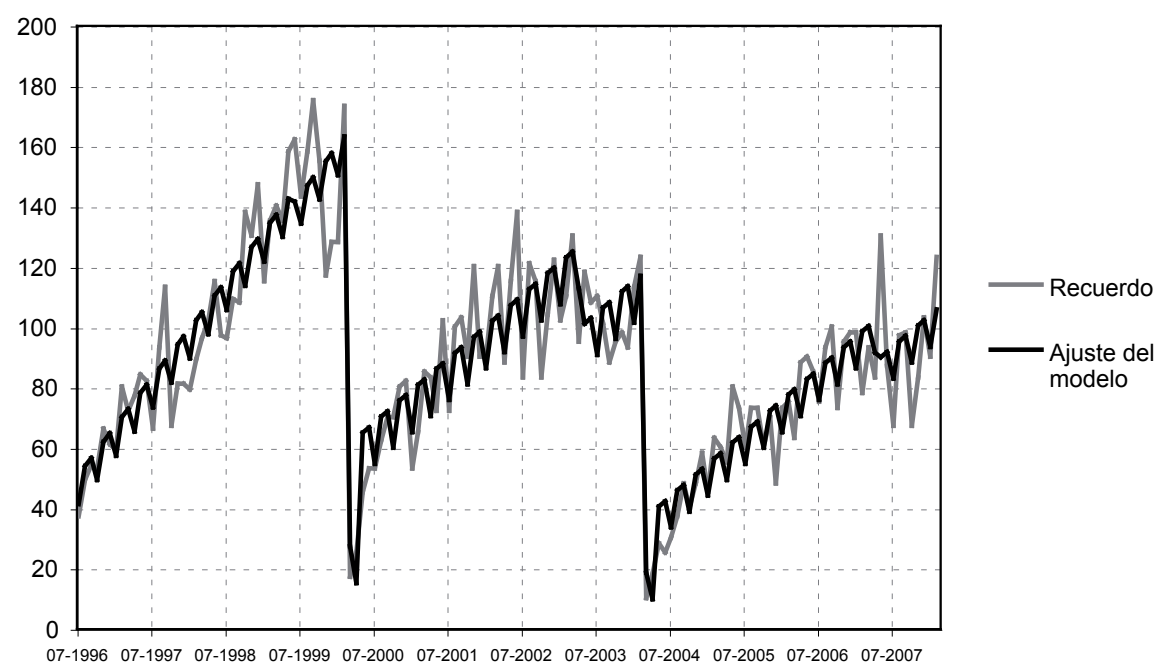

Fuente: Elaboración propia a partir del Barómetro del CIS, datos del Ministerio del Interior y del INE.

Además, propusimos que al alejarnos de la elección de referencia la probabilidad de obtener un "No recuerda" sería mayor (H6). Efectivamente, esta categoría comienza cada legislatura con valores cercanos a cero, experimentando un crecimiento sostenido en la dirección esperada.

\footnotetext{
${ }^{14}$ Resultados no mostrados.
} 
Este comportamiento del 'No recuerda' tiene una consecuencia muy concreta para nuestro análisis, ya que su crecimiento, conforme avanza la legislatura, afecta a la probabilidad de señalar cualquier otra categoría de respuesta. El Modelo Esperado incorpora el impacto del crecimiento progresivo de los nuevos electores y de las bajas en el censo, pero es ajeno a la incidencia del "No recuerda" sobre el resto de categorías de respuesta. Para aclarar este punto hemos calculado de nuevo las diferencias entre las pendientes después de incorporar el impacto de un "No recuerda" creciente (tabla 8). Hecho esto, tan sólo la moderada pendiente negativa del recuerdo al PSOE en 19962000 deja de mostrarse sesgada respecto al Modelo Esperado, no alterándose ninguna de las restantes conclusiones. Eso sí, queda claro que cualquier contraste que hagamos entre recuerdo de voto y resultados oficiales debe incorporar el impacto de un 'No recuerda' creciente sobre las demás categorías de respuesta.

Tabla 8.

Contraste entre las pendientes del Modelo Esperado y del

Barómetro del CIS tras incorporar el impacto del "No recuerda"

\begin{tabular}{lcc|cc|cc|cc}
\hline & \multicolumn{2}{c}{ No votó } & \multicolumn{2}{c}{ PP } & \multicolumn{2}{c}{ PSOE } & \multicolumn{2}{c}{ IU } \\
\hline & Esperado & CIS & Esperado & CIS & Esperado & CIS & Esperado & CIS \\
\hline $1996-2000$ & $-0,83$ & $+1,55^{* *}$ & $-1,14$ & $-0,84$ & $-1,10$ & $-1,46$ & $-0,31$ & $-2,04^{* *}$ \\
\hline $2000-2004$ & $-0,93$ & $-0,16^{* *}$ & $-0,96$ & $-2,54^{*}$ & $-0,74$ & $+2,41^{* *}$ & $-0,12$ & $-0,13$ \\
\hdashline $2004-2007$ & $-0,74$ & $+1,50^{* *}$ & $-0,94$ & $-1,02$ & $-1,07$ & $-2,68^{* *}$ & $-0,12$ & $+0,50$ \\
\hline
\end{tabular}

** La diferencia es significativa al 99\% ( $p>0,01)$.

* La diferencia es significativa al 95\% ( $p>0,05)$.

Fuente: Elaboración propia a partir del Barómetro del CIS, datos del Ministerio del Interior y del INE.

En otro orden, nuestra principal previsión respecto a los efectos de orden señalaba que en las encuestas electorales encontraríamos descensos significativos del "No contesta" (H5). Pues bien, dichos descensos se presentan en todas las legislaturas y con una elevada intensidad (gráfico 12). 
Gráfico 12.

Modelo para el recuerdo de voto "No Contesta"

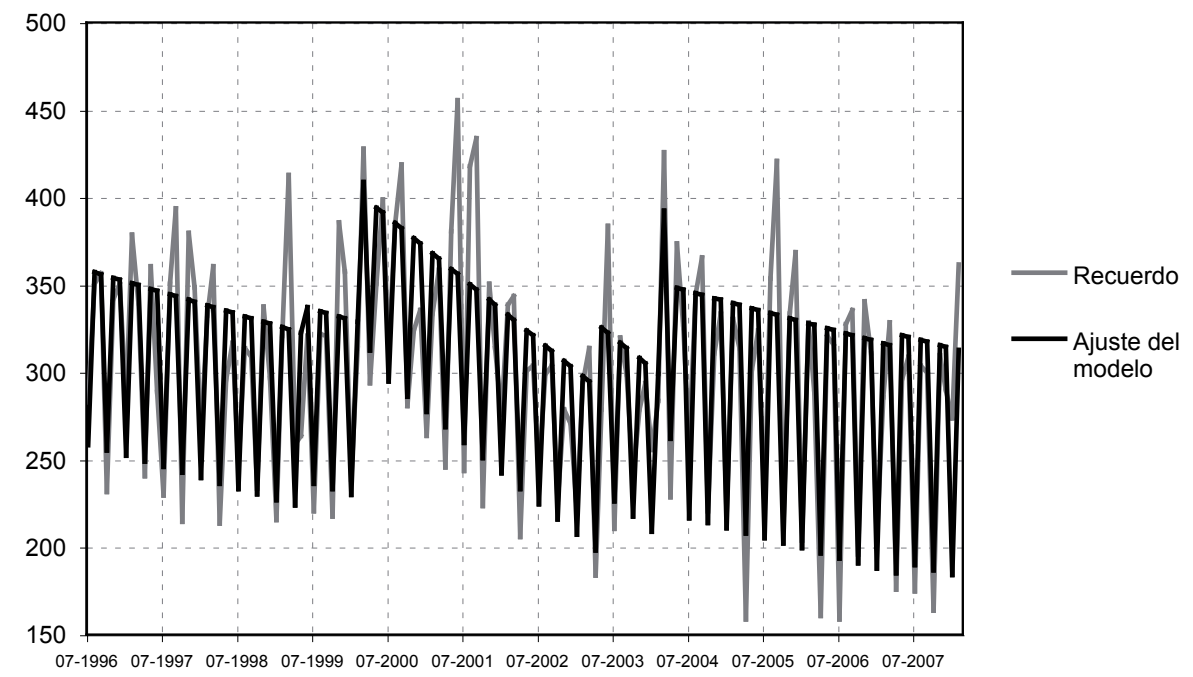

Fuente: Elaboración propia a partir del Barómetro del CIS.

Estas reducciones del 'No contesta' repercuten de manera desigual en las restantes categorías de respuesta. Como puede observarse en la tabla 1 sus mayores efectos se traducen en incrementos del recuerdo al PP en 2000-2004, y del PSOE en 2004-2007, períodos en que eran los partidos más votados. Por tanto, las encuestas electorales inducirían a sectores de la población a expresar sus adhesiones fundamentalmente en torno a las opciones mayoritarias.

Pero también encontramos otra pauta singular: junto a los descensos del 'No contesta', se reduce a su vez el recuerdo de abstención, lo que corroboraría la presencia de un sesgo de consistencia: desciende el temor a reconocer las preferencias partidarias — con caídas en el "No contesta"-y, al tiempo, se incrementa el interés por el tema del cuestionario y la probabilidad de dar una respuesta consistente, disminuyendo la vinculación al "No votó".

La evolución del "No contesta" presenta además una persistente pendiente negativa en los tres períodos. Las razones de esta evolución no resultan claras aunque, lógicamente, se relacionan con movimientos de signo contrario en las demás categorías de respuesta. Así, debemos tener cuenta el crecimiento del "No recuerda", el impacto de los nuevos electores y la evolución positiva del "No votó" en 1996-2000 y 2004-2008, y de "Votó al PSOE" entre 2000 y 2004. 
Gráfico 13.

Residuos para el recuerdo de voto PP

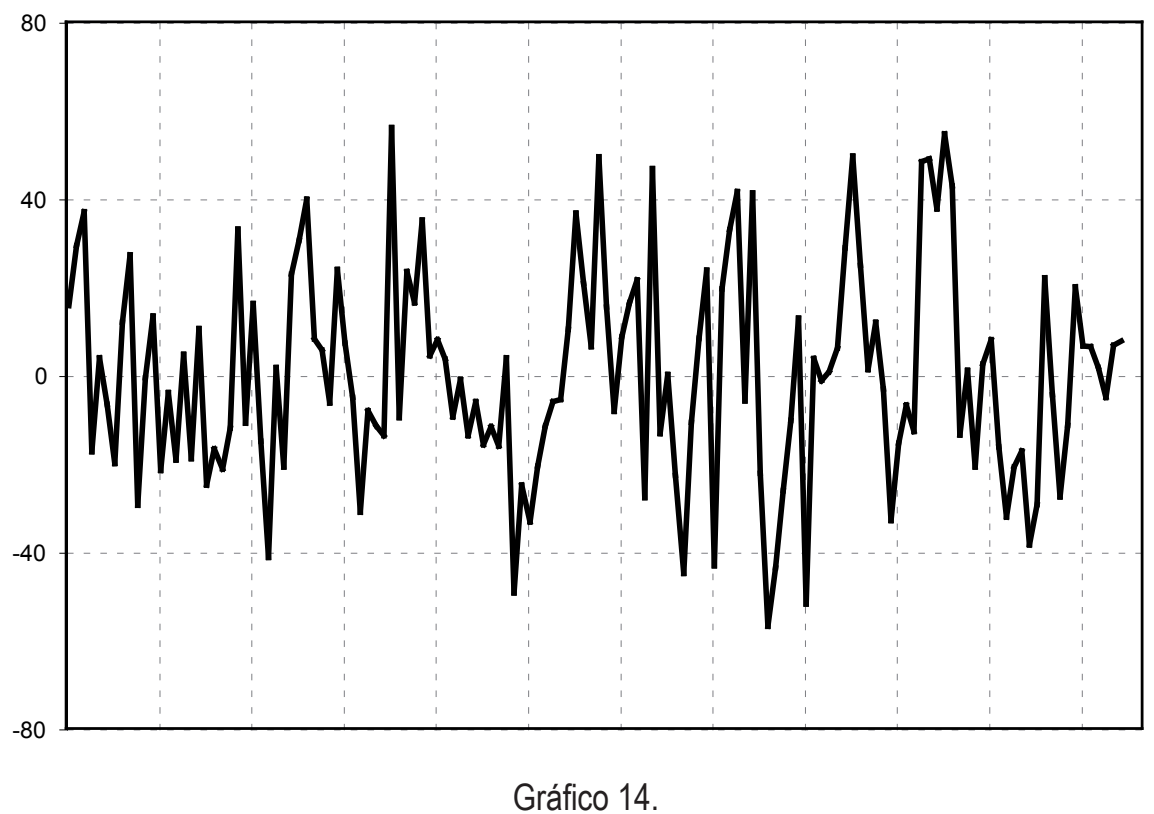

Residuos para el recuerdo de voto PSOE

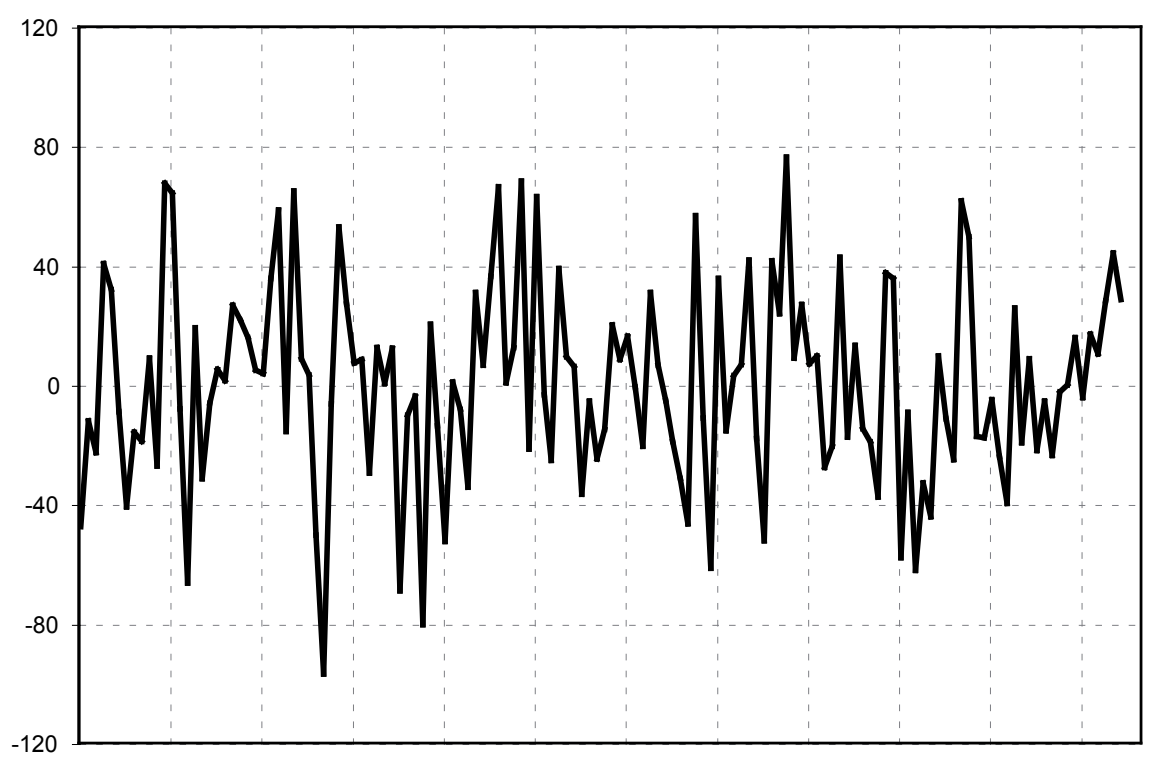

Fuente: Elaboración propia a partir del Barómetro del CIS. 


\section{Análisis final: los residuos ${ }^{15}$}

Finalmente, procedemos a una última revisión del trabajo atendiendo a los residuos de los modelos de regresión. Los residuos son las diferencias entre las puntuaciones pronosticadas y las realmente obtenidas en el Barómetro. Residuos positivos indican que hubo más entrevistados con recuerdo de voto para esa categoría de respuesta. Residuos negativos nos informan de lo contrario; que el número de entrevistados en la categoría resultó menor del esperado (gráficos 13 y 14).

Existe la posibilidad de que determinados eventos hayan perjudicado o favorecido a determinadas categorías de respuesta en el corto plazo. La imagen de algún partido pudo verse afectada con la suficiente intensidad como para que parte de los electores se desvinculara o se asociara al mismo temporalmente. Así que hemos procedido a contrastar si los residuos más destacados coinciden con eventos de la actualidad política que afectaron a la imagen de los partidos.

Pues bien, buena parte de los residuos coinciden con coyunturas muy relevantes. Residuos importantes para el PSOE se producen en momentos álgidos de sus crisis de liderazgo internas: noviembre de 1998, mayo de 1999 y abril y junio de 2000. Las declaraciones de tregua de ETA coinciden con residuos positivos del PSOE - septiembre de 1998, febrero de 2004 y marzo de 2006 - y la ruptura en enero de 2000 con un residuo negativo y un incremento para los populares. En 2004-2007, los residuos más elevados se producen en momentos clave del conflicto PP-PSOE por el Plan Ibarretxe (enero de 2005) y el Estatut (septiembre-noviembre de 2005 y enero-marzo de 2006). Sin embargo, en ocasiones los residuos más elevados no se corresponden con momentos de destacada conflictividad política —especialmente en la legislatura 2000-2004_, ni tampoco encontramos explicaciones para el importante residuo negativo del PSOE en marzo de 1999.

Los residuos elevados pueden tener otras causas. Por ejemplo, encontramos una mayor concentración en los barómetros electorales. En concreto, son los meses de julio los que experimentan una mayor proporción de desviaciones. Ya hemos mencionado que los problemas de participación en la encuesta pueden afectar gravemente a nuestra variable de análisis. En concreto, en los meses de julio de 2002, de 2004, de 2005 y de 2006, la incidencia de viviendas vacías experimentó incrementos importantes. Estos meses coinciden con residuos en el recuerdo. Sin embargo, las correspondencias no son precisas para otros meses de julio. Respecto a las negativas, las correspondencias son todavía más imprecisas, coincidiendo ocasionalmente con la presencia de residuos sin una pauta reconocible.

\footnotetext{
${ }^{15}$ Para establecer qué sectores sociales son más susceptibles de presentar un recuerdo de voto sesgado necesitaríamos un diseño de panel que indague en el recuerdo de los mismos individuos en dos momentos temporales. El examen de los perfiles sociodemográficos realizado no arroja resultados concluyentes (resultados no mostrados).
} 
Esta situación evidencia la necesidad de indagar en los efectos de las incidencias en la representatividad de las muestras. La entrevista personal es una modalidad cada día más compleja y en los meses de verano las dificultades para contactar son particularmente graves. Por tanto, podríamos mostrarnos más escépticos ante sesgos puntuales en los meses de julio y septiembre. Pero no podemos ir más allá y, en principio, parece que las distorsiones del recuerdo de voto se conducen por un camino relativamente independiente de las incidencias, excepto cuando estas adquieren una especial gravedad.

\section{CONCLUSIONES}

Como hemos visto, el recuerdo de voto estaría sesgado desde el comienzo mismo de cada legislatura. A partir de ahí, con el paso del tiempo se introducen nuevos sesgos, como los asociados al cambio en las preferencias electorales, al impacto del "No recuerda" y a la interferencia de las elecciones locales y regionales. En consecuencia, el recuerdo obtenido al comienzo de una legislatura, aunque contenga errores, resultará de mayor calidad que el encontrado años más tarde, pues al menos estará libre de estos sesgos adicionales. Además, si bien es cierto que trabajamos con un recuerdo sesgado, también lo es que un bloque - aparentemente sólido— de encuestados declararía consistentemente su voto, lo que apunta a un impacto limitado en los estudios de comportamiento electoral, probablemente exagerando o infravalorando algunos coeficientes.

A la hora de estimar la intención de voto y la fidelidad de los electores deberemos considerar que, pasados cuatro años desde la elección anterior, la distribución de un recuerdo de voto distorsionado podrá sesgar las estimaciones. Así, cuando una opción "pierde" durante una legislatura más recuerdo de voto del que debiera, es muy posible que terminemos sobrestimando la fidelidad de sus electores, ya que al final de la legislatura consideraremos a los sectores que han mantenido su recuerdo consistentemente. Posiblemente sea esto lo que ocurrió en los preelectorales del CIS con el voto a IU en 2000 y con el PP en 2004, para los que se estimó un grado de apoyo superior al finalmente obtenido. Al contrario, cuando una opción "crece" en recuerdo de voto, estaremos incluyendo a entrevistados que se han adherido progresivamente a la misma y, como resultado, terminaremos infraestimando sus apoyos, tal y como sucedió con el voto al PSOE en el preelectoral de 2004. Por tanto, antes de determinar la fidelidad de los electores será necesario considerar previamente estas evoluciones sesgadas. Sin embargo, mientras persista la sistemática infraestimación del electorado del Partido Popular, la práctica de emplear el recuerdo para estimar la intención de voto podría considerarse - hasta cierto punto y tomando las debidas precauciones- razonable.

Finalmente, este trabajo sugiere la importancia de aportar mayor información sobre el perfil de los que rechazan las encuestas políticas en España. Además, está a nuestro alcance analizar mediante un diseño de panel la variabilidad del recuerdo de voto, así como de otros elementos estratégicos como la autoubicación ideológica o la identificación partidaria. Un diseño de este tipo sí que nos permitiría determinar qué colectivos 
son más susceptibles de ofrecer un recuerdo sesgado, así como los patrones de cambio de otros indicadores relevantes, que muy probablemente son más inestables de lo que parecen. Así, las dificultades que afloren podrán ser afrontadas por el conocimiento de sus limitaciones y por las estrategias de mejora que promoverán.

\section{RefERENCIAS BibLIOGRÁfICAS}

Abelson, Robert P., Elisabeth F. Loftus y Anthony G. Greenwald. 1992. "Attempts to improve the accuracy of self-reports of voting." Pp.138-153 en Questions about Survey Questions: Meaning, Memory, Expression, and Social Interactions in Surveys, editado por J. Tanur. Nueva York: Russell Sage.

Adamany, David y Philip Dubois. 1975. "The "Forgetful" Voter and an Underreported Vote." The Public Opinion Quarterly 39: 227-231.

Andersson, Hans E. y Donald Granberg. 1997. "On the Validity and Reliability of Self-reported Vote: Validity Without Reliability?" Quality \& Quantity 31: 127-140.

Anderson, Barbara A. y Brian D. Silver. 1986. "Measurement and Mismeasurement of the Validity of the Self-reported Vote." American Journal of Political Science 30: 771-785.

Atkeson, Lonna R. 1999. "'Sure, I Voted for the Winner!" Overreport of the Primary Vote for the Party Nominee in the National Election Studies." Political Behavior 21: 197-215.

Barnett, Julie. 1998. "Sensitive questions and response effects: an evaluation." Journal of Managerial Psychology 13: 63-76.

Barton, Allen H. 1958. "Asking the Embarrassing Question." The Public Opinion Quarterly 22: 67-68.

Beckett, Megan, Julie Da Vanzo, Narayan Sastry, Constantijn Panis y Christine Peterson. 2001. "The Quality of Retrospective Data: An Examination of Long-Term Recall in a Developing Country." The Journal of Human Resources 36: 593-625.

Belli, Robert F., Michael W. Traugott y Matthew N. Beckmann. 2001. "What leads to Voting Overreports? Contrast of Overreporters to Validated Voters and Admitted Nonvoters in the American National Election Studies." Journal of Official Statistics 17: 479-498.

Belli, Robert F., Michael W. Traugott, Margaret Young y Katherine A. McGonagle. 1999. "Reducing Vote Overreporting in Surveys: Social Desirability, Memory Failure, and Source Monitoring." The Public Opinion Quarterly 63: 90-108.

Belli, Robert F., Sean E. Moore y John VanHoewyk. 2006. "An experimental comparison of question forms used to reduce vote overreporting." Electoral Studies 25: 751-759.

Benewick, Robert, Anthony H. Birch, Jay Blumler y Alison Ewbank. 1969. "The Floating Voter and the Liberal View of Representation." Political Studies 17: 177-195. 
Bernstein, Robert, Anita Chadha y Robert Montjoy. 2001. "Overreporting Voting: Why it Happens and Why it Matters." The Public Opinion Quarterly 65: 22-44.

Bradburn, Norman M. y William M. Mason. 1964. "The effect of question order on responses." Journal of Marketing Research 1: 57-61.

Bradburn, Norman M., Seymour Sudman y Brian Wansink. 2004. Asking Questions: the definitive guide to questionnaire design for market research, political polls, and social and health questionnaires. San Francisco: Jossey-Bass.

Brehm, John. 1993. The Phantom Respondents: Opinion Surveys and Political Representation. Ann Arbor: University of Michigan Press.

Calahan, Don. 1968. "Correlates of Respondent Accuracy in the Denver Validity Survey." The Public Opinion Quarterly 32: 607-621.

Campbell, Angus, Philip E. Converse, Warren E. Miller y Donald E. Stokes. 1980. The American Voter. Chicago: The University of Chicago Press.

Clausen, Aage. 1968. "Response Validity: Vote Report." The Public Opinion Quarterly 32: 588-606.

Crespi, Irving y Dwight Morris. 1984. "Question Order Effect and the Measurement of Candidate Preference in the 1982 Connecticut Elections." The Public Opinion Quarterly 48: 578-591.

Díaz de Rada, Vidal. 2008. "La selección de los entrevistados últimos en encuestas presenciales: un análisis de la utilización conjunta del método de rutas y el método de cuotas" REIS: Revista Española de Investigaciones Sociológicas 123: 209-247.

Díaz de Rada, Vidal y Adoración Núñez-Villuendas. 2008. Estudio de las Incidencias en la Investigación con Encuesta. El caso de los barómetros del CIS. Colección Monografías 251. Madrid: Centro de Investigaciones Sociológicas.

Dinerman, Helen. 1949. "1948 Votes in the Making -A Preview" The Public Opinion Quarterly 12: 585598.

Duff, Brian, Michael J. Hanmer, Won-ho Park y Ismail K. White. 2007. "Good Excuses: Understanding Who Votes with an Improved Turnout Question." The Public Opinion Quarterly 71: 67-90.

Eubank, Robert B. y David J. Gow. 1983. "The Pro-Incumbent Bias in the 1978 and 1980 National Election Studies." American Journal of Political Science 27: 122-139.

Freeman, Howard E. 1953. "A Note on the Prediction of Who Votes." The Public Opinion Quarterly 17: 288-292.

Gow, David J. y Robert B. Eubank. 1984. "The Pro-Incumbent Bias in the 1982 National Election Study." American Journal of Political Science 28: 224-230.

Granberg, Donald y Soren Holmberg. 1991. "Self-Reported Turnout and Voter Validation." American Journal of Political Science 35: 448-459. 
Granberg, Donald y Soren Holmberg. 1986. "Prior Behavior, Recalled Behavior, and the Prediction of Subsequent Voting Behavior in Sweden and the U.S." Human Relations 39: 135-148.

Grice, H. Paul. 1975. "Logic and conversation." Pp. 41-58 en Syntax and semantics, vol 3, editado por P. Cole. y J. Morgan. Nueva York: Academic Press.

Gronke, Paul. 1992. "Overreporting the Vote in the 1988 Senate Election Study: A Response to Wright." Legislative Studies Quarterly 17: 113-129.

Groves, Robert M., Stanley Presser. y Sarah Dipko. 2004. "The Role of Topic Interest in Survey Participation Decisions." The Public Opinion Quarterly 68: 2-31.

Himmelweit, Hilde T., Marianne J. Biberian y Janet Stockdale. 1978. "Memory for past Vote: Implications of a Study of Bias in Recall." British Journal of Political Science 8: 365-375.

Hochstim, Joseph R. y Dilman M. Smith. 1948. "Area Sampling or Quota Control?-Three Sampling Experiments." The Public Opinion Quarterly 12: 73-80.

Holbrook, Allyson L., Melanie C. Green y Jon A. Krosnick. 2003. "Telephone versus face-to-face interviewingof national probability samples with long questionnaires: Comparisons of respondent satisfying and social desirability response bias." The Public Opinion Quarterly 67: 79-125.

Holbrook, Allyson L. y Jon A. Krosnick. 2010. "Social desirability bias in voter turnout reports. Test using the item count technique." The Public Opinion Quarterly 74: 37-67.

Jackman, Simon. 1999. "Correcting Surveys for Non-Response and Measurement Error Using Auxiliary Information." Electoral Studies 18: 7-27.

Joslyn, Mark R. 2003. "The Determinants and Consequences of Recall Error about Gulf War Preferences." American Journal of Political Science 47: 440-452.

Kalton, Graham, Martin Collins y Lindsay Brook. 1978. "Experiments in Wording Opinion Questions." Applied Statistics 27: 149-161.

Kalton, Graham y Howard Schuman. 1982. "The Effect of the Question on Survey Responses: A Review." Journal of the Royal Statistical Society. Series A 145: 42-73.

Karp, Jeffrey A. y Susan Banducci. 1999. "The Impact of Proportional Representation on Turnout: Evidence from New Zeland." Australian Journal of Political Science 34: 363-377.

Karp, Jeffrey A. y David Brockington. 2005. "Social Desirability and Response Validity: A Comparative Analysis of Overreporting Voter Turnout in Five Countries." The Journal of Politics 67: 825-840.

Katosh, John P. y Michael W. Traugott. 1981. "The consequences of Validated and self-reported voting measures." The Public Opinion Quarterly 45: 519-535.

Katz, Richard S., Richard G. Niemi y David Newman. 1980. "Reconstructing Past Partisianship in Britain." Bristish Journal of Political Science 10: 505-537.

Kruskal, William y Frederick Mosteller. 1980. "Representative Sampling, IV: The History of the Concept in Statistics." International Statistical Review 48: 169-195. 
Levine, Linda J. 1997. "Reconstructing memory for emotions." Journal of Experimental Psychology 126: 165-177.

Lynn, Peter. 1995. "Evidence of the inaccuracy of quota samples." Survey Methods Centre Newsletter 15: 20-23.

Lynn, Peter y Roger Jowell. 1996. "How Might Opinion Polls be Improved?: The Case for Probability Sampling." Journal of the Royal Statistical Society. Series A 159: 21-28.

Marcus, Gregory B. 1986. "Stability and change in political attitudes: Observe, recall, and "explain."” Political Behavior 8: 21-44.

Mason, Robert, Virginia Lesser y Michael W. Traugott. 2002. "Effect of Item Nonresponse on Nonresponse Error and Inference." pp.149-161 en Survey Nonresponse, editado por R. Groves et al. New York: Wiley-Interscience.

McDermid, Robert H. 1989. "The Recall of Past Partisanship: Feeble Memories or Frail Concepts?" Canadian Journal of Political Science 22: 363-375.

McDonald, Hugh E. y Edward R. Hirt. 1997. "When Expectancy Meets Desire: Motivational Effects in Reconstructive Memory." Journal of Personality and Social Psychology 72: 5-23.

McFarland, Sam G. 1981. "Effects of Question Order on Survey Responses." The Public Opinion Quarterly 45: 208-215.

Miller, Mungo. 1952. "The Waukegan Study of Voter Turnout Prediction." The Public Opinion Quarterly 16: 381-398.

Moon, Nick. 1995. "The case for quota sampling." Survey Methods Centre Newsletter 15: 9-12.

Moore, David W. 2002. "Measuring New Types of Question-Order Effects: Additive and Subtractive." The Public Opinion Quarterly 66: 80-91.

Moser, Claus y Alan Stuart. 1953. "An Experimental Study of Quota Sampling." Journal of the Royal Statistical Society. Series A 116: 349-405.

Niemi, Richard G., Richard S. Katz y David Newman. 1980. "Reconstructing Past Partisanship: The Failure of the Party Identification Recall Questions." American Journal of Political Science 24: 633651.

Noelle-Neumann, Elisabeth. 1995. La espiral del silencio. Opinión pública: nuestra piel social. Barcelona: Paidós Ibérica.

Noelle-Neumann, Elisabeth. 1970. "Wanted: Rules for Wording Structured Questionnaires." The Public Opinion Quarterly 34: 191-201.

Núñez-Villuendas, Adoración. 2005. "Incidencias de la Entrevista Personal en la investigación mediante Encuesta." Revista Española de Investigaciones Sociológicas 109: 219-236. 
Parry, Hugh J. y Helen M. Crossley. 1950. "Validity of Responses to Survey Questions." The Public Opinion Quarterly 14: 61-80.

Plumb, Elizabeth. 1986. "Validation of Voter Recall: Time of Electoral Decision Making." Political Behavior 8: $302-312$.

Presser, Stanley. 1984. "Is Inaccuracy on Factual Survey Items Item-Specific or Respondent-Specific?" The Public Opinion Quarterly 48: 344-355.

Presser, Stanley y Michael Traugott. 1992. "Little white lies and social science models: Correlated response errors in a panel Study of Voting." The Public Opinion Quarterly 56: 77-86.

Schacter, Daniel L. 1999. "The Seven Sins of Memory: Insights From Psychology and Cognitive Neuroscience." American Psychologist 54: 182-203.

Schacter, Daniel L. y Chad S. Dodson. 2001. "Misattribution, False Recognition and the Sins of Memory." Philosophical Transactions of the Royal Society - Biological Sciences 356: 1385-1393.

Schacter, Daniel L., Joan Y. Chiao y Jason P. Mitchell. 2003. "The Seven Sins of Memory: Implications for Self." Annals New York Academy of Sciences 1001: 226-239.

Schaeffer, Nora C. y Stanley Presser. 2003. "The science of asking questions." Annual Review of Sociology 29: 65-88.

Schuman, Howard y Stanley Presser. 1996. Questions and Answers in Attitudes Surveys. Thousand Oaks: Sage.

Scheufele, Dietram A. y Patricia Moy. 2000. "Twenty-five years of the spiral of silence: a conceptual review and empirical outlook." International Journal of Public Opinion Research 12: 3-28.

Shaw, Daron, Rodolfo De la Garza y Jongho Lee. 2000. "Examining Latino Turnout in 1996: A ThreeState, Validated Survey Approach." American Journal of Political Science 44: 332-340.

Shoemaker, Pamela J., Martin Eichholz y Elizabeth A. Skewes. 2000. "Item Nonresponse: Distinguishing Between Don"t Know and Refuse." International Journal of Public Opinion Research 14: 193201.

Sigelman, Lee. 1982. "The nonvoting voter in voting research." American Journal of Political Science 26: $47-56$.

Sigelman, Lee. 1981. "Question-Order Effects on Presidential Popularity." The Public Opinion Quarterly 45: 199-207.

Silver, Brian D., Paul R. Abramson y Barbara A. Anderson. 1986. "The presence of others and Overreporting of Voting in American National Elections." The Public Opinion Quarterly 50: 228-239.

Silver, Brian D., Barbara A. Anderson y Paul R. Abramson. 1986. "Who Overreports Voting?." American Political Science Review 80: 613-624.

Smith, Tom W. 1984. "Recalling Attitudes: An Analysis of Retrospective Questions on the 1982 GSS." The Public Opinion Quarterly 48: 639-649. 
Smith, Tom W. 1983. "On the Validity of Inferences from Non-random Sample." Journal of the Royal Statistical Society. Series A 146: 394-403.

Stephenson, C. Bruce. 1979. "Probability Sampling With Quotas: An Experiment." The Public Opinion Quarterly 43: 477-496.

Stocké, Volker. 2007. "Response Privacy and Elapsed Time Since Election Day as Determinants for Vote Overreporting." International Journal of Public Opinion Research 19: 237-246.

Stocké, Volker y Tobias Stark. 2007. "Political Involvement and Memory Failure as Interdependent Determinants of Vote Overreporting." Applied Cognitive Psychology 21: 239-257.

Sudman, Seymour. 1966. "Probability Sampling with Quotas." Journal of the American Statistical Association 61: 749- 771 .

Swaddle, Kevin y Anthony Heath. 1989. "Official and Reported Turnout in the British General Election of 1987." British Journal of Political Science 19: 537-570.

Tourangeau, Roger y Ting Yan. 2007. "Sensitive Questions in Surveys." Psychological Bulletin 133: 859-883.

Traugott, Michael W. y John P. Katosh. 1979. "Response validity in surveys of voting behavior." The Public Opinion Quarterly 43: 359-377.

Urquizu, Ignacio. 2005. "El Voto Oculto en España." Revista Española de Ciencia Política 13: 119-156.

Van der Eijk, Cees y Broer Niemöller. 1983. "Recall Accuracy and its Determinants." Acta Politica 14: 289-342.

Voogt, Robert J. 2005. "An alternative Approach to Correcting Response and Nonresponse Bias in Election Research" Acta Politica 40: 94-116.

Voogt, Robert J. y Hetty Van Kempen. 2002. "Nonresponse Bias and Stimulus Effects in the Dutch National Election Study." Quality \& Quantity 36: 325-345.

Waldhal, Ragnar y Bernt Aardal. 2000. "The accuracy of recalled previous voting: evidence from Norwegian Election Study Panels." Scandinavian Political Studies 23: 373-389.

Waldhal, Ragnar y Bernt Aardal. 1982. "Can We Trust Recall-Data?" Scandinavian Political Studies 5: 101-116.

Weir, Blair T. 1975. "The distortion of voter recall." American Journal of Political Science 19: 53-62.

Weisberg, Herbert F. 2005. The Total Survey Error Approach. Chicago: The University of Chicago Press.

Wright, Gerald C. 1993. "Errors in Measuring Vote Choice in the National Election Studies, 1952-88." American Journal of Political Science 37: 291-316.

Wright, Gerald C. 1992. "Reported Versus Actual Vote: There is a Difference and it Matters." Legislative Studies Quarterly 17: 131-142. 
Wright, Gerald C. 1990. "Misreports of Vote Choice in the 1988 NES Senate Election Study." Legislative Studies Quarterly 15: 543-563.

Wu, Chung-li. 2006. "Vote misreporting and survey context: the Taiwan case." Issues \& Studies 42: 223-239.

JAIME BALAGUER es Licenciado en Sociología por la UNED y magíster en Democracia y Gobierno por la Universidad Autónoma de Madrid. Desde 2007 es Titulado Superior del Centro de Investigaciones Sociológicas (CIS). Sus áreas de interés principal son la metodología de encuesta y el análisis de indicadores electorales.

RECIBIDO: $17 / 10 / 2008$

ACEPTADO: 23/04/2009 
Tabla A1.

Descriptivos del recuerdo de voto entre julio de 1996 y febrero de 2008 en el Barómetro del CIS (en número de entrevistados)

\begin{tabular}{|c|c|c|c|c|}
\hline & & VI Lesgislatura & VII Legislatura & VIII Legislatura \\
\hline \multirow{4}{*}{ IU } & Mínimo & 90 & 82 & 62 \\
\hline & Máximo & 221 & 118 & 109 \\
\hline & Media & 155 & 98 & 89 \\
\hline & Desv. Tip. & 32,8 & 9,5 & 11,1 \\
\hline \multirow{4}{*}{ PP } & Mínimo & 552 & 534 & 430 \\
\hline & Máximo & 721 & 832 & 592 \\
\hline & Media & 629 & 677 & 493 \\
\hline & Desv. Tip. & 42,9 & 70,1 & 37,7 \\
\hline \multirow{4}{*}{ PSOE } & Mínimo & 480 & 470 & 764 \\
\hline & Máximo & 731 & 683 & 1093 \\
\hline & Media & 616 & 578 & 861,9 \\
\hline & Desv. Tip. & 51,9 & 49,5 & 77,5 \\
\hline \multirow{4}{*}{ No votó } & Mínimo & 269 & 311 & 262 \\
\hline & Máximo & 427 & 490 & 481 \\
\hline & Media & 347 & 420 & 398 \\
\hline & Desv. Tip. & 34,6 & 41,7 & 42,2 \\
\hline \multirow{4}{*}{ No tenía edad } & Mínimo & 14 & 0 & 0 \\
\hline & Máximo & 178 & 143 & 118 \\
\hline & Media & 100 & 77 & 57 \\
\hline & Desv. Tip. & 47,2 & 40,4 & 33,8 \\
\hline $\mathrm{N}$ & & 40 & 44 & 44 \\
\hline
\end{tabular}

Fuente: Elaboración propia a partir de Barómetro del CIS. 


\section{Gráficos A1.}

Contraste entre el Recuerdo del Barómetro del CIS y el recuerdo en los estudios del ISSP*, Latinobarómetro y preelectorales del CIS

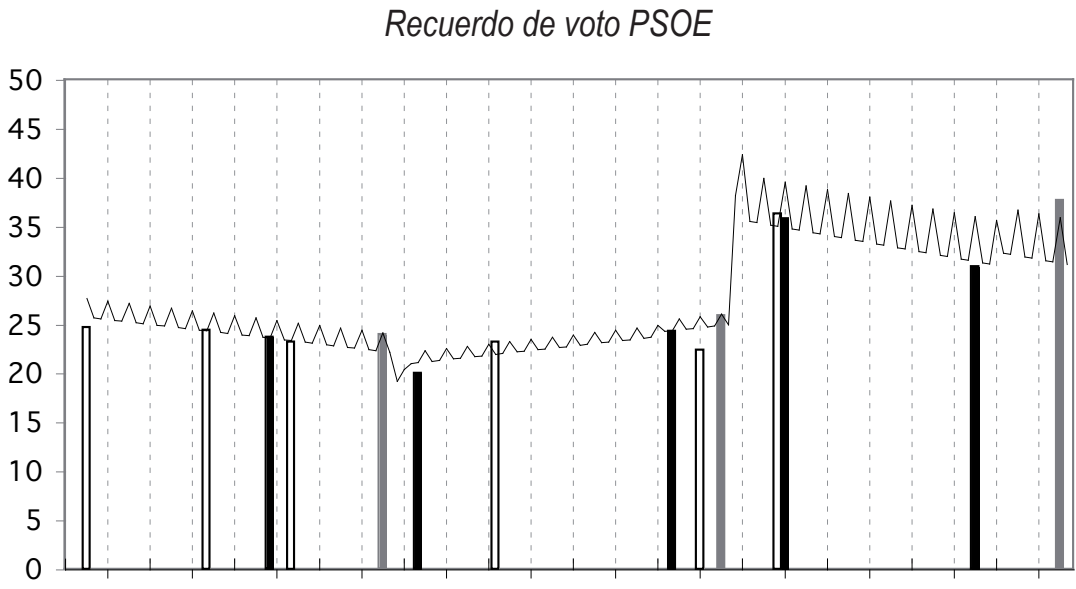

199619971998199920002001200220032004200520062007

$\square$ ISSP $\square$ Latinobarómetro Preelectorales CIS — Barómetro CIS

\section{Recuerdo de voto PP}

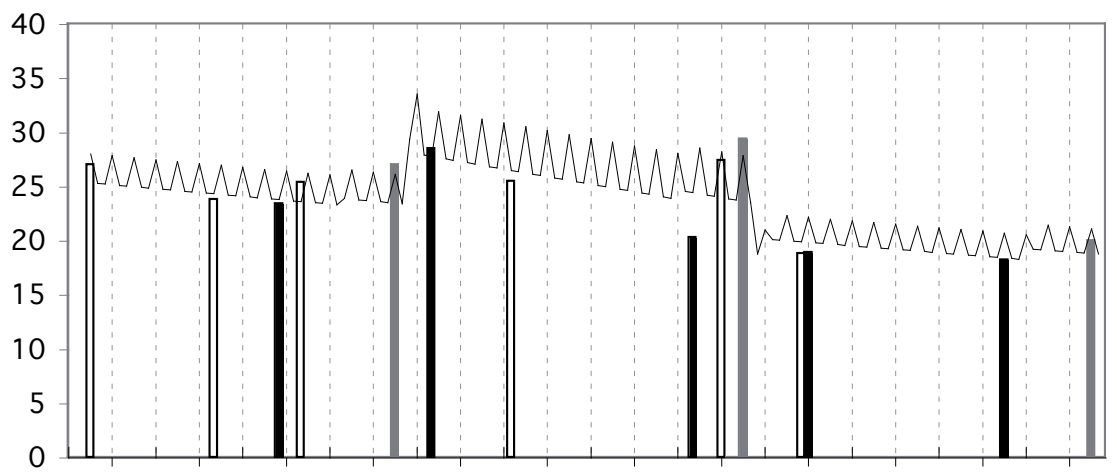

199619971998199920002001200220032004200520062007

$\square$ ISSP $\square$ Latinobarómetro $\square$ Preelectorales CIS — Barómetro CIS

* Los estudios del ISSP de 2000 y 2007 fueron completamente probabilísticos. 
Gráficos A1.

Continuación

Recuerdo de voto IU

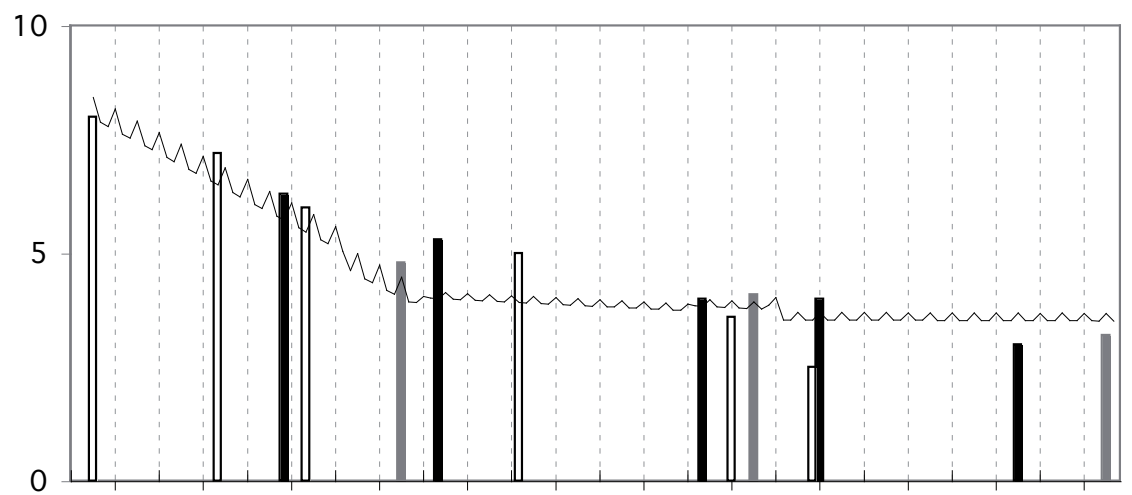

199619971998199920002001200220032004200520062007

$\square$ ISSP $\square$ Latinobarómetro Preelectorales CIS — Barómetro CIS

Recuerdo de No votó

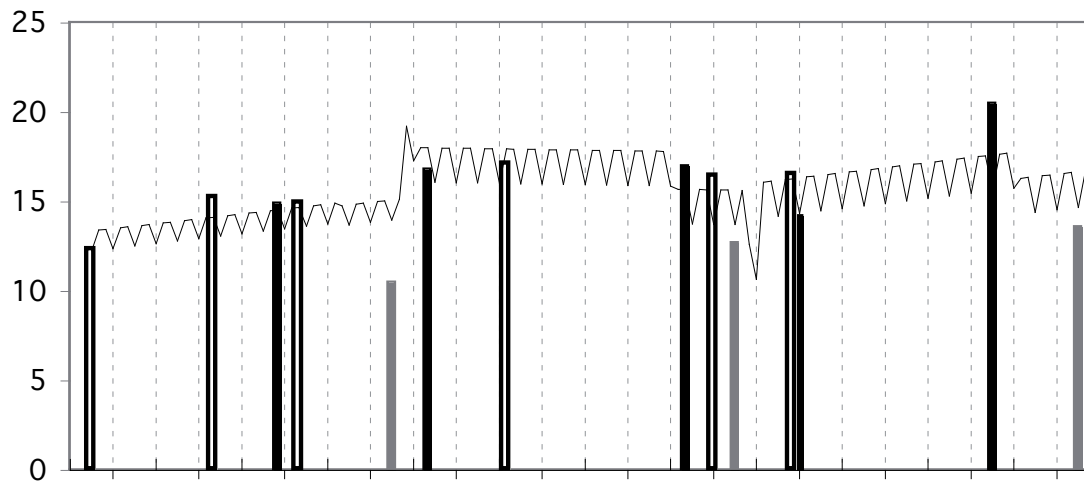

199619971998199920002001200220032004200520062007

$\square$ ISSP $\square$ Latinobarómetro $\quad$ Preelectorales CIS — Barómetro CIS 